\title{
Global Existence of Generalized Solutions of the Spherically Symmetric Einstein-Scalar Equations in the Large
}

\author{
Demetrios Christodoulou* \\ Departments of Mathematics and Physics, Syracuse University, Syracuse, New York, USA
}

\begin{abstract}
In this paper we study the global initial value problem for the spherically symmetric Einstein-scalar field equations in the large. We introduce the concept of a generalized solution of our problem, and, taking as initial hypersurface a future light cone with vertex at the center of symmetry, we prove, without any restriction on the size of the initial data, the global, in retarded time, existence of generalized solutions.
\end{abstract}

\section{Section 0. Introduction}

In [1] we began the study of the global initial value problem for Einstein's equations in the spherically symmetric case with a massless scalar field as the material model. In terms of a radial coordinate $r$ and a retarded time coordinate $u$, whose level surfaces are future light cones with vertices at the center of symmetry, the spacetime metric has the form

$$
d s^{2}=-e^{2 v} d u^{2}-2 e^{v+\lambda} d u d r+r^{2} d \Sigma^{2},
$$

where $d \Sigma^{2}$ is the metric of the standard 2-sphere. We reduced Einstein's equations to a single nonlinear evolution equation for the function $h=\partial(r \phi) / \partial r$, where $\phi$ is the matter field. If $f$ is a function of $u$ and $r$ we denote by $\bar{f}$ the mean value function of $f$ :

$$
\bar{f}(u, r):=(1 / r) \int_{0}^{r} f\left(u, r^{\prime}\right) d r^{\prime}
$$

Then, letting

$$
g:=\exp \left[-4 \pi \int_{r}^{\infty}(h-\bar{h})^{2} \frac{d r}{r}\right]
$$

\footnotetext{
* Research supported in part by National Science Foundation grants MCS-8201599 to the Courant Institute and PHY-8318350 to Syracuse University
} 
and

$$
D:=\frac{\partial}{\partial u}-\frac{1}{2} \bar{g} \frac{\partial}{\partial r}
$$

the nonlinear evolution equation is:

$$
D h=\frac{1}{2 r}(g-\bar{g})(h-\bar{h}) .
$$

If $h$ is a classical solution of this equation, then setting $e^{\nu+\lambda}=g, e^{\nu-\lambda}=\bar{g}$ and $\phi=\bar{h}$, the above metric together with the matter field $\phi$ satisfy Einstein's equations

$$
R_{\mu \nu}=8 \pi \partial_{\mu} \phi \partial_{\nu} \phi
$$

The integral curves of $D$ are the incoming light rays. The initial data of our problem is the function $h$ at $u=0$. In [1] we proved, for general initial data, the local, in retarded time, existence of a classical solution (Theorem 1 of [1]). We also proved that if the initial data is sufficiently small, there exists a global classical solution which disperses in the infinite future (Theorem 3 of [1]). In this paper we shall study the global problem for arbitrarily large initial data. Such data will lead to gravitational collapse. Now, there may not in general exist a classical solution for all retarded time.

In the field of hydrodynamics we have the Navier-Stokes equations describing the motion of a viscous incompressible fluid. Also there, we can prove the global existence of a classical solution only if the Reynold's number of the initial data is sufficiently small. But for large initial data a classical solution may exist only for a short time. This situation lead Leray in his fundamental work of 1934 [2] to introduce the concept of a generalized solution of the initial value problem of hydrodynamics. He called such a solution "a turbulent solution" and he proved that for arbitrary initial data of finite kinetic energy there exists for all time at least one turbulent solution.

In the present paper we shall introduce an appropriate concept of generalized solution for the mathematical model we are considering. This model differs from hydrodynamics in being of hyperbolic rather than parabolic character. Also, in our problem the spacetime itself is constructed from the solution. In [1] we defined the mass $m(u, r)$ enclosed within the sphere of radius $r$ at retarded time $u$ by

$$
m:=(r / 2)(1-\bar{g} / g) \text {. }
$$

The total (Bondi) mass $M(u)$ at retarded time $u$ is then given by $M(u):=\lim _{r \rightarrow \infty} m(u, r)$. One of the main difficulties of our problem is that the total mass $M$ does not provide an estimate for the solution in the interior of the sphere of radius $2 M$. Another main difficulty stems from the fact that one of the Einstein equations is the local mass equation:

$$
D m=-\pi \xi^{2} / g, \text { where } \xi:=2 r D \bar{h} .
$$

It is therefore necessary to require a generalized solution to satisfy not only the nonlinear evolution equation for $h$, but also the evolution equation for the local mass $m$. (This requirement is automatically satisfied for classical solutions.) 
The principal tool of our investigation is what we call the main integral identity. Letting $\chi_{u_{1}}\left(u ; r_{1}\right)$ denote the incoming light ray through $r=r_{1}$ at $u=u_{1}$ and setting

$$
Q\left(u_{1}, r_{1}\right):=\left\{(u, r) \mid 0<u<u_{1}, 0<r<\chi_{u_{1}}\left(u ; r_{1}\right)\right\},
$$

the main integral identity is given by:

$$
\int_{0}^{r_{1}} \frac{g}{\bar{g}}\left(u_{1}, r\right) d r+2 \pi \iint_{Q\left(u_{1}, r_{1}\right)} \frac{g \xi^{2}}{\bar{g}^{2} r} d r d u+\frac{1}{2} \int_{0}^{u_{1}} g(u, 0) d u=\int_{0}^{r_{0}} \frac{g}{\bar{g}}(0, r) d r,
$$

where $r_{0}:=\chi_{u_{1}}\left(0 ; r_{1}\right)$. The main integral identity equates the sum of three positive definite integrals, the first of which is an integral over the future light cone $u=u_{1}$, the second a spacetime integral, and the third an integral over the central line, to an integral over the initial future light cone $u=0$. The quantity in the integral over the future light cone is $\left(1-\frac{2 m}{r}\right)^{-1}$. As we shall see, this integral provides the necessary estimate for the solution in the interior. The spacetime integral provides an additional estimate which is essential in showing that the generalized solution satisfies the local mass equation. It should be noted that the main integral identity, in contrast to the mass-flux relation, holds only in the domain of outer communications.

The plan of this paper is the following. In Sect. 1 we define what we shall mean by generalized solution and we state the global existence theorem (Theorem 1); Sects. 2-5 are devoted to the proof of this theorem. In Sect. 2 we introduce a regularization of the nonlinear evolution equation depending on a positive parameter $\varepsilon$, and we derive the corresponding local mass equation and the integral identities which follow. The $\varepsilon$-regularized problem is in fact the original problem in the presence of a white hole of mass $\varepsilon / 2$ and with the boundary condition that the scalar field vanishes on the anti-event horizon. In Sect. 3 we prove the global existence and uniqueness of classical solutions of the $\varepsilon$-regularized evolution equation. In Sect. 4 we derive various $\varepsilon$-independent estimates for these solutions. In Sect. 5 we study the limit $\varepsilon \rightarrow 0$ of removing the white hole, making various compactness arguments which lead to the proof of Theorem 1.

In a subsequent paper we shall study the structure and uniqueness of generalized solutions.

\section{Section I. Generalized Solutions and the Global Existence Theorem}

Let us be given initial data $h_{0}(r)$ in $C^{1}[0, \infty[$ and such that the initial Bondi mass $M_{0}$ is finite. Let $Q$ denote the complement of the central line: $Q:=\{(u, r), 0 \leqq u<\infty, 0<r<\infty\}$.

Definition. A global generalized solution of the problem is a function $h \in C^{1}(Q)$ such that at each $u, h$ belongs to $L^{2}(0, \infty)$ and $\int_{0}^{\infty} h^{2} d r$ is bounded by a continuous function of $u$, having the following properties. $h$ satisfies the nonlinear evolution equation $D h=\frac{1}{2 r}(g-\bar{g})(h-\bar{h})$ in $Q, \bar{h}, g$ and $\bar{g}$ being continuous in $Q$, and $h(0, r)$ 
$=h_{0}(r)$. Also, at each $u, g / \bar{g}$ belongs to $L^{1}\left(0, r_{0}\right), r_{0}$ arbitrary. Furthermore, for almost all $u$,

$$
\xi:=\lim _{\delta \rightarrow 0} \int_{\delta}^{r} \bar{g}(h-\bar{h}) \frac{d r}{r}
$$

exists and $g^{1 / 2} \xi / \bar{g} r^{1 / 2} \in L^{2}\left(\left(0, u_{0}\right) \times\left(0, r_{0}\right)\right), u_{0}, r_{0}$ arbitrary. In addition, $\bar{h}$ is weakly differentiable in $Q$ and $D \bar{h}=\frac{\xi}{2 r}$, and $m$ is weakly differentiable in $Q$ and $D m$ $=-\frac{\pi}{g} \xi^{2}$. Finally, for each $\left(u_{1}, r_{1}\right) \in Q$, the main integral identity

$$
\int_{0}^{r_{1}} \frac{g}{\bar{g}}\left(u_{1}, r\right) d r+2 \pi \iint_{Q\left(u_{1}, r_{1}\right)} \frac{g \xi^{2}}{\bar{g}^{2} r} d r d u+\frac{1}{2} \int_{0}^{u_{1}} g(u, 0) d u=\int_{0}^{r_{0}} \frac{g}{\bar{g}}(0, r) d r
$$

holds, where $Q\left(u_{1}, r_{1}\right)=\left\{(u, r) \mid 0<r<\chi_{u_{1}}(u ; r), 0<u<u_{1}\right\}$.

All the conditions of the above definition are needed in order to have a meaningful solution of Einstein's equations. The purpose of this paper is the proof of:

Theorem 1. For each initial data $h_{0} \in C^{1}[0, \infty$ [ of finite initial Bondi mass, there exists at least one global generalized solution of the problem.

\section{Section 2. The Regularization Method}

We shall now give a regularization of the nonlinear evolution equation depending on a positive parameter $\varepsilon$. The regularization method is based on following redefinition of the mean value operation defined in [1], Sect. 3. If $f_{\varepsilon}$ is a function of $u$ and $r$ depending on $\varepsilon$, we set:

$$
\overline{f_{\varepsilon}}(u, r):=\frac{1}{r+\varepsilon} \int_{0}^{r} f_{\varepsilon}\left(u, r^{\prime}\right) d r^{\prime}
$$

We then have

$$
f_{\varepsilon}=\frac{\partial}{\partial r}\left((r+\varepsilon) \overline{f_{\varepsilon}}\right) \quad \text { or } \quad \frac{\partial \overline{f_{\varepsilon}}}{\partial r}=\frac{\left(f_{\varepsilon}-\overline{f_{\varepsilon}}\right)}{r+\varepsilon} .
$$

We note that $\bar{f}_{\varepsilon}(u, 0)=0$. The principal unknown function shall now be denoted by $h_{\varepsilon}$, and $\bar{h}_{\varepsilon}$ shall be given by

$$
\overline{h_{\varepsilon}}:=\frac{1}{r+\varepsilon} \int_{0}^{r} h_{\varepsilon} d r
$$

We also define

$$
A_{\varepsilon}:=\int_{r}^{\infty}\left(h_{\varepsilon}-\overline{h_{\varepsilon}}\right)^{2} \frac{d r}{r+\varepsilon}
$$

and we set

$$
g_{\varepsilon}:=e^{-4 \pi A_{\varepsilon}}
$$


and

$$
\bar{g}_{\varepsilon}:=\frac{1}{r+\varepsilon} \int_{0}^{r} g_{\varepsilon} d r
$$

We note that $\bar{g}_{\varepsilon} \leqq g_{\varepsilon}$. Denoting then by $D_{\varepsilon}$ the differential operator,

$$
D_{\varepsilon}:=\frac{\partial}{\partial u}-\frac{1}{2} \bar{g}_{\varepsilon} \frac{\partial}{\partial r}
$$

the $\varepsilon$-regularized evolution equation is:

$$
D_{\varepsilon} h_{\varepsilon}=\frac{1}{2(r+\varepsilon)}\left(g_{\varepsilon}-\bar{g}_{\varepsilon}\right)\left(h_{\varepsilon}-\bar{h}_{\varepsilon}\right) \text {. }
$$

The characteristics of this equation shall be denoted by $\chi_{\varepsilon}$. They satisfy the ordinary differential equation,

$$
\frac{d r}{d u}=-\frac{1}{2} \bar{g}_{\varepsilon}
$$

We note that, since $\bar{g}_{\varepsilon}(u, 0)=0$ and $\partial \bar{g}_{\varepsilon} / \partial r$ is bounded by $1 / \varepsilon$, the $\varepsilon$-characteristic through any point on the central line is the central line itself.

We now define the $\varepsilon$-local mass function by:

$$
m_{\varepsilon}=\frac{(r+\varepsilon)}{2}\left(1-\bar{g}_{\varepsilon} / g_{\varepsilon}\right)
$$

We have $m_{\varepsilon}(u, 0)=\varepsilon / 2$ and $m_{\varepsilon}<(r+\varepsilon) / 2$ for $r>0$. Also,

$$
\frac{\partial m_{\varepsilon}}{\partial r}=\frac{(r+\varepsilon)}{2} \frac{\bar{g}_{\varepsilon}}{g_{\varepsilon}^{2}} \frac{\partial g_{\varepsilon}}{\partial r}=2 \pi \frac{\bar{g}_{\varepsilon}}{g_{\varepsilon}}\left(h_{\varepsilon}-\bar{h}_{\varepsilon}\right)^{2} .
$$

Thus $m_{\varepsilon}$ is a monotonically nondecreasing function of $r$ at each $u$, bounded from below by $\varepsilon / 2$, and we can write

$$
m_{\varepsilon}=\frac{\varepsilon}{2}+2 \pi \int_{0}^{r} \frac{\bar{g}_{\varepsilon}}{g_{\varepsilon}}\left(h_{\varepsilon}-\overline{h_{\varepsilon}}\right)^{2} d r .
$$

We also define the $\varepsilon$-total mass

$$
M_{\varepsilon}(u):=\lim _{r \rightarrow \infty} m_{\varepsilon}(u, r) .
$$

We shall derive the evolution law of $\bar{h}_{\varepsilon}, A_{\varepsilon}$ and $m_{\varepsilon}$ along the characteristics $\chi_{\varepsilon}$. We have

$$
\begin{aligned}
D_{\varepsilon} \bar{h}_{\varepsilon}= & \frac{1}{r+\varepsilon} \int_{0}^{r} \frac{\partial h_{\varepsilon}}{\partial u} d r-\frac{1}{2} \bar{g}_{\varepsilon} \frac{\partial \bar{h}_{\varepsilon}}{\partial r}=\frac{1}{r+\varepsilon} \int_{0}^{r}\left(D_{\varepsilon} h_{\varepsilon}+\frac{1}{2} \bar{g}_{\varepsilon} \frac{\partial h_{\varepsilon}}{\partial r}\right) d r \\
& -\frac{1}{2(r+\varepsilon)} \bar{g}_{\varepsilon}\left(h_{\varepsilon}-\bar{h}_{\varepsilon}\right) .
\end{aligned}
$$

Setting then

$$
D_{\varepsilon} \bar{h}_{\varepsilon}=\frac{\xi_{\varepsilon}}{2(r+\varepsilon)}
$$


and using the $\varepsilon$-regularized evolution Eq. (2.7), we obtain:

$$
\begin{aligned}
\xi_{\varepsilon} & =\int_{0}^{r}\left(2 D_{\varepsilon} h_{\varepsilon}+\bar{g}_{\varepsilon} \frac{\partial h_{\varepsilon}}{\partial r}\right) d r-\bar{g}_{\varepsilon}\left(h_{\varepsilon}-\bar{h}_{\varepsilon}\right)=\int_{0}^{r}\left[\frac{\partial \bar{g}_{\varepsilon}}{\partial r}\left(h_{\varepsilon}-\bar{h}_{\varepsilon}\right)+\bar{g}_{\varepsilon} \frac{\partial h_{\varepsilon}}{\partial r}\right] d r-\bar{g}_{\varepsilon}\left(h_{\varepsilon}-\bar{h}_{\varepsilon}\right) \\
& =-\left[\bar{g}_{\varepsilon}\left(h_{\varepsilon}-\bar{h}_{\varepsilon}\right)\right]_{r=0}+\int_{0}^{r} \bar{g}_{\varepsilon} \frac{\partial \bar{h}_{\varepsilon}}{\partial r} d r,
\end{aligned}
$$

and since $\bar{g}_{\varepsilon}(u, 0)=0$, we conclude that

$$
\xi_{\varepsilon}=\int_{0}^{r} \bar{g}_{\varepsilon}\left(h_{\varepsilon}-\bar{h}_{\varepsilon}\right) \frac{d r}{r+\varepsilon} .
$$

In particular, $\xi_{\varepsilon}(u, 0)=0$. From $(2.3)$ we obtain

$$
\begin{aligned}
D_{\varepsilon} A_{\varepsilon}= & \int_{r}^{\infty} 2\left(h_{\varepsilon}-\bar{h}_{\varepsilon}\right)\left[\frac{1}{2} \bar{g}_{\varepsilon} \frac{\partial h_{\varepsilon}}{\partial r}-\frac{1}{2(r+\varepsilon)} \bar{g}_{\varepsilon}\left(h_{\varepsilon}-\bar{h}_{\varepsilon}\right)+D_{\varepsilon} h_{\varepsilon}-D_{\varepsilon} \bar{h}_{\varepsilon}\right] \frac{d r}{r+\varepsilon} \\
& +\frac{1}{2(r+\varepsilon)} \bar{g}_{\varepsilon}\left(h_{\varepsilon}-\bar{h}_{\varepsilon}\right)^{2} .
\end{aligned}
$$

Using then (2.7) we find

$$
(r+\varepsilon) \frac{\partial}{\partial r} D_{\varepsilon} A_{\varepsilon}=-\frac{1}{2(r+\varepsilon)} g_{\varepsilon}\left(h_{\varepsilon}-\bar{h}_{\varepsilon}\right)^{2}+2\left(h_{\varepsilon}-\bar{h}_{\varepsilon}\right) D_{\varepsilon} \bar{h}_{\varepsilon}
$$

Taking into account the fact that $D_{\varepsilon} r=-\bar{g}_{\varepsilon} / 2$, we obtain from (2.9):

$$
D_{\varepsilon} m_{\varepsilon}=-\frac{1}{4} \bar{g}_{\varepsilon}\left(1-\bar{g}_{\varepsilon} / g_{\varepsilon}\right)+\frac{(r+\varepsilon)}{2} \frac{\bar{g}_{\varepsilon}}{g_{\varepsilon}}\left(\frac{D_{\varepsilon} g_{\varepsilon}}{g_{\varepsilon}}-\frac{D_{\varepsilon} \bar{g}_{\varepsilon}}{\bar{g}_{\varepsilon}}\right) \text {. }
$$

Now, by (2.4): $D_{\varepsilon} g_{\varepsilon}=-4 \pi g_{\varepsilon} D_{\varepsilon} A_{\varepsilon}$ and

$$
D_{\varepsilon} \bar{g}_{\varepsilon}=\frac{1}{(r+\varepsilon)} \int_{0}^{r}\left(D_{\varepsilon} g_{\varepsilon}+\frac{1}{2} \bar{g}_{\varepsilon} \frac{\partial g_{\varepsilon}}{\partial r}\right) d r-\frac{1}{2(r+\varepsilon)} \bar{g}_{\varepsilon}\left(g_{\varepsilon}-\bar{g}_{\varepsilon}\right) .
$$

Substituting in (2.15) we obtain:

$$
D_{\varepsilon} m_{\varepsilon}=2 \pi(r+\varepsilon) \frac{\bar{g}_{\varepsilon}}{g_{\varepsilon}}\left[-D_{\varepsilon} A_{\varepsilon}+\frac{1}{(r+\varepsilon) \bar{g}_{\varepsilon}} \int_{0}^{r} D_{\varepsilon} A_{\varepsilon} g_{\varepsilon} d r+\frac{1}{(r+\varepsilon) \bar{g}_{\varepsilon}} \int_{0}^{r} \frac{1}{2} \bar{g}_{\varepsilon} g_{\varepsilon} \frac{\partial A_{\varepsilon}}{\partial r} d r\right] .
$$

On the other hand, for general $f$ it holds:

$$
\frac{1}{(r+\varepsilon) \bar{g}_{\varepsilon}} \int_{0}^{r} f g_{\varepsilon} d r-f=-\frac{1}{(r+\varepsilon) \bar{g}_{\varepsilon}} \int_{0}^{r}(r+\varepsilon) \bar{g}_{\varepsilon} \frac{\partial f}{\partial r} d r
$$

Hence

$$
D_{\varepsilon} m_{\varepsilon}=-\frac{2 \pi}{g_{\varepsilon}} \int_{0}^{r} \bar{g}_{\varepsilon}\left[(r+\varepsilon) \frac{\partial D_{\varepsilon} A_{\varepsilon}}{\partial r}-\frac{1}{2} g_{\varepsilon} \frac{\partial A_{\varepsilon}}{\partial r}\right] d r .
$$

By (2.14), (2.3), and (2.12), (2.13) the integrant is:

$$
\bar{g}_{\varepsilon}\left[(r+\varepsilon) \frac{\partial D_{\varepsilon} A_{\varepsilon}}{\partial r}-\frac{1}{2} g_{\varepsilon} \frac{\partial A_{\varepsilon}}{\partial r}\right]=2 \bar{g}_{\varepsilon}\left(h_{\varepsilon}-\bar{h}_{\varepsilon}\right) D_{\varepsilon} \bar{h}_{\varepsilon}=\xi_{\varepsilon} \frac{\partial \xi_{\varepsilon}}{\partial r} .
$$


Therefore, since $\xi_{\varepsilon}(u, 0)=0$, we conclude that the evolution law of $m_{\varepsilon}$ along the characteristics $\chi_{\varepsilon}$ is

$$
D_{\varepsilon} m_{\varepsilon}=-\frac{\pi}{g_{\varepsilon}} \xi_{\varepsilon}^{2}
$$

Integrating this equation along the $\varepsilon$-characteristic $\chi_{\varepsilon, u_{1}}\left(u ; r_{1}\right)$ through $r=r_{1}$ at $u=u_{1}$, we obtain the $\varepsilon$-mass-flux relation:

$$
m_{\varepsilon}\left(u_{1}, r_{1}\right)=m_{\varepsilon}\left(0, r_{0, \varepsilon}\right)-\pi \int_{0}^{u_{1}}\left[\xi_{\varepsilon}^{2} / g_{\varepsilon}\right]_{\chi_{\varepsilon}, u_{1}}\left(u_{1} ; r_{1}\right) d u
$$

where $r_{0, \varepsilon}=\chi_{\varepsilon, u_{1}}\left(0 ; r_{1}\right)$ is the value of $r$ at which this characteristic intersects $r=0$.

$$
\begin{aligned}
& \text { Since } g_{\varepsilon} / \bar{g}_{\varepsilon}=\left(1-\frac{2 m_{\varepsilon}}{r+\varepsilon}\right)^{-1},(2.18) \text { implies that } \\
& \begin{aligned}
D_{\varepsilon}\left(g_{\varepsilon} / \bar{g}_{\varepsilon}\right) & =2\left(1-\frac{2 m_{\varepsilon}}{r+\varepsilon}\right)^{-2}\left(-\frac{\pi}{(r+\varepsilon)} \frac{\xi_{\varepsilon}^{2}}{g_{\varepsilon}}+\frac{1}{2} \bar{g}_{\varepsilon} \frac{m_{\varepsilon}}{(r+\varepsilon)^{2}}\right) \\
& =-2 \pi \frac{g_{\varepsilon}}{\bar{g}_{\varepsilon}^{2}} \frac{\xi_{\varepsilon}^{2}}{(r+\varepsilon)}+\frac{1}{2} \frac{g_{\varepsilon}}{\bar{g}_{\varepsilon}} \frac{\partial \bar{g}_{\varepsilon}}{\partial r} .
\end{aligned}
\end{aligned}
$$

Let $\delta$ be a fixed positive real number. We have

$$
\begin{aligned}
D_{\varepsilon}\left(\int_{\delta}^{r} \frac{g_{\varepsilon}}{\bar{g}_{\varepsilon}} d r\right) & =\int_{\delta}^{r} \frac{\partial}{\partial u}\left(\frac{g_{\varepsilon}}{\bar{g}_{\varepsilon}}\right) d r-\frac{1}{2} g_{\varepsilon}=\int_{\delta}^{r}\left[D_{\varepsilon}\left(\frac{g_{\varepsilon}}{\bar{g}_{\varepsilon}}\right)+\frac{1}{2} \bar{g}_{\varepsilon} \frac{\partial}{\partial r}\left(\frac{g_{\varepsilon}}{\bar{g}_{\varepsilon}}\right)\right] d r-\frac{1}{2} g_{\varepsilon} \\
& =\int_{\delta}^{r}\left[D_{\varepsilon}\left(\frac{g_{\varepsilon}}{\bar{g}_{\varepsilon}}\right)-\frac{1}{2} \frac{\partial \bar{g}_{\varepsilon}}{\partial r}\left(\frac{g_{\varepsilon}}{\bar{g}_{\varepsilon}}\right)\right] d r-\frac{1}{2}\left[g_{\varepsilon}\right]_{r=\delta} .
\end{aligned}
$$

Thus, by (2.20):

$$
D_{\varepsilon}\left(\int_{\delta}^{r} \frac{g_{\varepsilon}}{\bar{g}_{\varepsilon}} d r\right)=-2 \pi \int_{\delta}^{r} \frac{g_{\varepsilon}}{\bar{g}_{\varepsilon}^{2}} \frac{\xi_{\varepsilon}^{2}}{(r+\varepsilon)} d r-\frac{1}{2}\left[g_{\varepsilon}\right]_{r=\delta} \ldots
$$

Integrating this equation along $\chi_{\varepsilon, u_{1}}\left(u ; r_{1}\right)$, we obtain the $\varepsilon$-integral identity:

$\int_{\delta}^{r_{1}}\left(\frac{g_{\varepsilon}}{\bar{g}_{\varepsilon}}\right)\left(u_{1}, r\right) d r+2 \pi \iint_{Q_{\delta, \varepsilon}\left(u_{1}, r_{1}\right)} \frac{g_{\varepsilon}}{\bar{g}_{\varepsilon}^{2}} \frac{\xi_{\varepsilon}^{2}}{(r+\varepsilon)} d r d u+\frac{1}{2} \int_{0}^{u_{1}} g_{\varepsilon}(u, \delta) d u=\int_{\delta}^{r_{0, \varepsilon}}\left(\frac{g_{\varepsilon}}{\bar{g}_{\varepsilon}}\right)(0, r) d r$,

where $Q_{\delta, \varepsilon}\left(u_{1}, r_{1}\right)=\left\{(u, r) \mid 0<u<u_{1}, \delta<r<\chi_{\varepsilon, u_{1}}\left(u ; r_{1}\right)\right\}$.

If $h_{\varepsilon}$ is a classical solution of (2.7), the spacetime which is the manifold $\mathbb{R} \times \mathbb{R}^{+}$ $\times S^{2}$ endowed with the metric $d s^{2}=-g_{\varepsilon} \bar{g}_{\varepsilon} d u^{2}-2 g_{\varepsilon} d u d r+(r+\varepsilon)^{2} d \Sigma^{2}$, together with the scalar field $\phi=\bar{h}_{\varepsilon}$ is in fact a classical solution of the original Einsteinscalar field equations $R_{\mu \nu}=8 \pi \partial_{\mu} \phi \partial_{\nu} \phi$. The boundary $\mathbb{R} \times[r=0] \times S^{2}$ is, in view of the fact that $\bar{g}_{\varepsilon}(u, 0)=0$, a past null cylinder, namely an anti-even horizon, of crosssectional area equal to $4 \pi \varepsilon^{2}$. Thus the $\varepsilon$-regularized problem is in fact the original problem in the presence of a white hole of a mass $m_{\varepsilon}(u, 0)=\varepsilon / 2$, and with the boundary condition that the scalar field vanishes on the anti-event horizon: $\bar{h}_{\varepsilon}(u, 0)=0$. 


\section{Section 3. Classical Solutions of the Regularized Problem}

In the following we shall assume that the initial data satisfies the falloff conditions $h_{0}=O\left(r^{-3}\right)$ and $\partial h_{0} / \partial r=O\left(r^{-4}\right)$ as $r \rightarrow \infty$ in order to obtain the fastest possible falloff at null infinity. The results easily extend to the case when we only assume that $M_{0}$ is finite.

Lemma 1. For every initial data $h_{0}(r) \in C^{1}\left[0, \infty\left[\right.\right.$ such that $h_{0}(r)=O\left(r^{-3}\right)$ and $\partial g_{0} / \partial r(r)=O\left(r^{-4}\right)$, and for each $\varepsilon>0$, there exists a unique global classical solution $h_{\varepsilon}(u, r) \in C^{1}[0, \infty[\times[0, \infty[$ of the $\varepsilon$-regularized evolution equation taking the given data at $u=0$, and such that at each $u \geqq 0, h_{\varepsilon}(u, r)=O\left(r^{-3}\right)$ and $\partial h_{\varepsilon} / \partial r(u, r)=O\left(r^{-4}\right)$ as $r \rightarrow \infty$.

Proof. First, we can prove the existence of a local classical solution by using the argument of Theorem 1 of [1]. To prove global existence we argue as follows: Let $\left[0, u_{0}\left[\right.\right.$ be the maximal interval of existence. We shall show that $u_{0}=\infty$. For if $u_{0}$ is finite, we shall demonstrate that $h_{\varepsilon}(u, r)$, which is by assumption a $C^{1}$-function on $\left[0, u_{0}\left[\times\left[0, \infty\left[\right.\right.\right.\right.$, can be extended to a $C^{1}$-function at $\left\{u_{0}\right\} \times[0, \infty[$. The local existence theorem with data at $u=u_{0}$ would then contradict the maximality of $u_{0}$.

We first derive global a priori bounds for $h_{\varepsilon}$ and $\partial h_{\varepsilon} / \partial r$. Setting

$$
x(u):=\sup _{r \geqq 0}\left|h_{\varepsilon}(u, r)\right|,
$$

we have

$$
\left|h_{\varepsilon}-\bar{h}_{\varepsilon}\right| \leqq 2 x
$$

and from (2.7):

$$
\left|D_{\varepsilon} h_{\varepsilon}\right| \leqq \frac{x}{\varepsilon} .
$$

Integrating this along an $\varepsilon$-characteristic we obtain

$$
\left|h_{\varepsilon}\left(u_{1}, r_{1}\right)\right| \leqq\left|h_{0}\left(r_{0, \varepsilon}\right)\right|+\int_{0}^{u_{1}} \frac{x(u)}{\varepsilon} d u .
$$

Hence

$$
x\left(u_{1}\right) \leqq x_{0}+\int_{0}^{u_{1}} \frac{x(u)}{\varepsilon} d u
$$

where

$$
x_{0}:=\sup _{r \geq 0}\left|h_{0}(r)\right|
$$

It follows that

$$
x\left(u_{1}\right) \leqq x_{0} e^{u_{1} / \varepsilon} .
$$

Differentiating now (2.7) with respect to $r$ we obtain:

$$
D_{\varepsilon}\left(\frac{\partial h_{\varepsilon}}{\partial r}\right)=\frac{\left(g_{\varepsilon}-\bar{g}_{\varepsilon}\right)}{(r+\varepsilon)} \frac{\partial h_{\varepsilon}}{\partial r}+\frac{1}{2(r+\varepsilon)^{2}}\left[-3\left(g_{\varepsilon}-\bar{g}_{\varepsilon}\right)+4 \pi g_{\varepsilon}\left(h_{\varepsilon}-\bar{h}_{\varepsilon}\right)^{2}\right]\left(h_{\varepsilon}-\bar{h}_{\varepsilon}\right) \text {. }
$$


We have $\left|-3\left(g_{\varepsilon}-\bar{g}_{\varepsilon}\right)+4 \pi g_{\varepsilon}\left(h_{\varepsilon}-\overline{h_{\varepsilon}}\right)^{2}\right| \leqq\left(3+16 \pi x^{2}\right)$. Setting then

$$
y(u):=\sup _{r \geqq 0}\left|\frac{\partial h_{\varepsilon}}{\partial r}(u, r)\right| \text {, }
$$

we obtain:

$$
\left|D_{\varepsilon}\left(\frac{\partial h_{\varepsilon}}{\partial r}\right)\right| \leqq \frac{1}{\varepsilon} y+\frac{x}{\varepsilon^{2}}\left(3+16 \pi x^{2}\right) \text {. }
$$

Integration along an $\varepsilon$-characteristic yields

$$
\left|\frac{\partial h_{\varepsilon}}{\partial r}\left(u_{1}, r_{1}\right)\right| \leqq\left|\frac{\partial h_{0}}{\partial r}\left(r_{0, \varepsilon}\right)\right|+\int_{0}^{u_{1}} \frac{y(u)}{\varepsilon} d u+\int_{0}^{u_{1}} \frac{x(u)}{\varepsilon^{2}}\left(3+16 \pi x^{2}(u)\right) d u .
$$

Hence

$$
y\left(u_{1}\right) \leqq y_{0}+\int_{0}^{u_{1}} \frac{y(u)}{\varepsilon} d u+\int_{0}^{u_{1}} \frac{x(u)}{\varepsilon^{2}}\left(3+16 \pi x^{2}(u)\right) d u
$$

where

$$
y_{0}:=\sup _{r \geqq 0}\left|\frac{\partial h_{0}}{\partial r}(r)\right| \text {. }
$$

It follows that

$$
y\left(u_{1}\right) \leqq e^{u_{1} / \varepsilon}\left[y_{0}+\frac{1}{\varepsilon^{2}} \int_{0}^{u_{1}} x(u)\left(3+16 \pi x^{2}(u)\right) d u\right] .
$$

We conclude that $h_{\varepsilon}$ and $\partial h_{\varepsilon} / \partial r$, and therefore also $\partial h_{\varepsilon} / \partial u$ are uniformly bounded in $\left[0, u_{0}\left[\times\left[0, \infty\left[\right.\right.\right.\right.$. To prove that $h_{\varepsilon}(u, r)$ can be extended to a $C^{1}$ function at $\left\{u_{0}\right\}$ $\times\left[0, \infty\left[\right.\right.$, we need to show that $\partial h_{\varepsilon} / \partial r$ is uniformly continuous in $\left[0, u_{0}[\times[0, \infty[\right.$. Actually, we need only show uniform continuity with respect to $r$. Uniform continuity with respect to $u$ would then follow by the following argument: From (3.9) and the a priori bounds, it follows that $D_{\varepsilon}\left(\partial h_{\varepsilon} / \partial r\right)$ is uniformly bounded:

$$
\left|D_{\varepsilon}\left(\partial h_{\varepsilon} / \partial r\right)\right| \leqq C \text {. }
$$

Given $u_{1}, r_{1}$ and $\Delta u_{1}>0$, let $r_{1}+\Delta r_{1, \varepsilon}$ be the value of $r$ at which the $\varepsilon$-characteristic through $\left(u_{1}, r_{1}\right)$ intersects the line $u=u_{1}-\Delta u_{1}$. Then $\Delta r_{1, \varepsilon} \leqq 1 / 2 \Delta u_{1}$, and we have

$$
\begin{aligned}
& \left|\frac{\partial h_{\varepsilon}}{\partial r}\left(u_{1}, r_{1}\right)-\frac{\partial h_{\varepsilon}}{\partial r}\left(u_{1}-\Delta u_{1}, r_{1}\right)\right| \leqq \int_{u_{1}-\Delta u_{1}}^{u_{1}}\left|D_{\varepsilon}\left(\partial h_{\varepsilon} / \partial r\right)\right| d u \\
& \quad+\left|\frac{\partial h_{\varepsilon}}{\partial r}\left(u_{1}-\Delta u_{1}, r_{1}+\Delta r_{1, \varepsilon}\right)-\frac{\partial h_{\varepsilon}}{\partial r}\left(u_{1}-\Delta u_{1}, r_{1}\right)\right| \\
& \leqq C \cdot \Delta u_{1}+\eta_{\varepsilon}\left(\Delta r_{1, \varepsilon}\right) \leqq C \cdot \Delta u_{1}+\eta_{\varepsilon}\left(\frac{1}{2} \Delta u_{1}\right),
\end{aligned}
$$

where $\eta_{\varepsilon}$ is the modulus of continuity of $\partial h_{\varepsilon} / \partial r$ with respect to $r$.

Let $0 \leqq r_{1}^{\prime}<r_{1}$, and let us denote by $\chi_{\varepsilon}\left(u ; r_{1}\right)$ and $\chi_{\varepsilon}\left(u ; r_{1}^{\prime}\right)$ the $\varepsilon$-characteristics through $r=r_{1}$ and $r=r_{1}^{\prime}$, respectively, at $u=u_{1}$. We set

$$
\psi_{\varepsilon}(u):=\frac{\partial h_{\varepsilon}}{\partial r}\left(u, \chi_{\varepsilon}\left(u ; r_{1}\right)\right)-\frac{\partial h_{\varepsilon}}{\partial r}\left(u, \chi_{\varepsilon}\left(u ; r_{1}^{\prime}\right)\right) \text {. }
$$


Then, from (3.7) we have

$$
\frac{d \psi_{\varepsilon}}{d u}=\left(\frac{\partial \bar{g}_{\varepsilon}}{\partial r} \frac{\partial h_{\varepsilon}}{\partial r}\right)_{\left(u, \chi_{\varepsilon}\left(u: r_{1}\right)\right)}-\left(\frac{\partial \bar{g}_{\varepsilon}}{\partial r} \frac{\partial h_{\varepsilon}}{\partial r}\right)_{\left(u, \chi_{\varepsilon}\left(u: r_{1}^{\prime}\right)\right)}+f_{\varepsilon}\left(u, \chi_{\varepsilon}\left(u ; r_{1}\right)\right)-f_{\varepsilon}\left(u, \chi_{\varepsilon}\left(u ; r_{1}^{\prime}\right)\right),
$$

where

$$
f_{\varepsilon}:=\frac{1}{2(r+\varepsilon)^{2}}\left[-3\left(g_{\varepsilon}-\bar{g}_{\varepsilon}\right)+4 \pi g_{\varepsilon}\left(h_{\varepsilon}-\bar{h}_{\varepsilon}\right)^{2}\right]\left(h_{\varepsilon}-\bar{h}_{\varepsilon}\right)
$$

We have

$$
\begin{aligned}
\frac{\partial f_{\varepsilon}}{\partial r}= & \frac{1}{2(r+\varepsilon)^{2}} \frac{\partial h_{\varepsilon}}{\partial r}\left[-3\left(g_{\varepsilon}-\bar{g}_{\varepsilon}\right)+12 \pi g_{\varepsilon}\left(h_{\varepsilon}-\bar{h}_{\varepsilon}\right)^{2}\right] \\
& +\frac{2}{(r+\varepsilon)^{3}}\left(h_{\varepsilon}-\bar{h}_{\varepsilon}\right)\left[3\left(g_{\varepsilon}-\bar{g}_{\varepsilon}\right)-8 \pi g_{\varepsilon}\left(h_{\varepsilon}-\bar{h}_{\varepsilon}\right)^{2}+4 \pi^{2} g_{\varepsilon}\left(h_{\varepsilon}-\bar{h}_{\varepsilon}\right)^{4}\right]
\end{aligned}
$$

Hence

$$
\sup _{r \geqq 0}\left|\frac{\partial f_{\varepsilon}}{\partial r}\right| \leqq \frac{1}{2 \varepsilon^{2}} y\left(3+48 \pi x^{2}\right)+\frac{2}{\varepsilon^{2}} x\left(3+32 \pi x^{2}+64 \pi^{2} x^{4}\right) .
$$

From the a priori bounds it then follows that $\partial f_{\varepsilon} / \partial r$ is uniformly bounded,

Therefore

$$
\sup _{r \geqq 0}\left|\frac{\partial f_{\varepsilon}}{\partial r}\right| \leqq b_{1}
$$

$$
\left|f_{\varepsilon}\left(u, \chi_{\varepsilon}\left(u ; r_{1}\right)\right)\right|-f_{\varepsilon}\left(u, \chi_{\varepsilon}\left(u ; r_{1}^{\prime}\right)\right) \mid \leqq b_{1}\left(\chi_{\varepsilon}\left(u ; r_{1}\right)-\chi_{\varepsilon}\left(u ; r_{1}^{\prime}\right)\right) .
$$

We write

We have

$$
\begin{aligned}
& \left(\frac{\partial \bar{g}_{\varepsilon}}{\partial r} \frac{\partial h_{\varepsilon}}{\partial r}\right)_{\left(u, \chi_{\varepsilon}\left(u ; r_{1}\right)\right)}-\left(\frac{\partial \bar{g}_{\varepsilon}}{\partial r} \frac{\partial h_{\varepsilon}}{\partial r}\right)_{\left(u, \chi_{\varepsilon}\left(u ; r_{1}^{\prime}\right)\right)}=\left(\frac{\partial \bar{g}_{\varepsilon}}{\partial r}\right)_{\left(u_{1}, \chi_{\varepsilon}\left(u ; r_{1}\right)\right)} \cdot \psi_{\varepsilon}(u) \\
& \quad+\left[\left(\frac{\partial \bar{g}_{\varepsilon}}{\partial r}\right)_{\left(u, \chi_{\varepsilon}\left(u ; r_{1}\right)\right)}-\left(\frac{\partial \bar{g}_{\varepsilon}}{\partial r}\right)_{\left(u, \chi_{\varepsilon}\left(u ; r_{1}^{\prime}\right)\right)}\right]\left(\frac{\partial h_{\varepsilon}}{\partial r}\right)_{\left(u, \chi_{\varepsilon}\left(u ; r_{1}^{\prime}\right)\right)} .
\end{aligned}
$$

$$
\frac{\partial^{2} \bar{g}_{\varepsilon}}{\partial r^{2}}=\frac{1}{(r+\varepsilon)^{2}}\left[4 \pi g_{\varepsilon}\left(h_{\varepsilon}-\bar{h}_{\varepsilon}\right)^{2}-2\left(g_{\varepsilon}-\bar{g}_{\varepsilon}\right)\right]
$$

Hence

$$
\sup _{r \geqq 0}\left|\frac{\partial^{2} \bar{g}_{\varepsilon}}{\partial r^{2}}\right| \leqq \frac{2}{\varepsilon^{2}}\left(8 \pi x^{2}+1\right) \text {. }
$$

Thus $\partial^{2} \bar{g}_{\varepsilon} / \partial r^{2}$ is uniformly bounded,

$$
\sup _{r \geqq 0}\left|\frac{\partial^{2} \bar{g}_{\varepsilon}}{\partial r^{2}}\right| \leqq b_{0}
$$

and therefore

$$
\left|\left(\frac{\partial \bar{g}_{\varepsilon}}{\partial r}\right)_{\left(u, \chi_{\varepsilon}\left(u ; r_{1}\right)\right)}-\left(\frac{\partial \bar{g}_{\varepsilon}}{\partial r}\right)_{\left(u, \chi_{\varepsilon}\left(u ; r_{1}^{\prime}\right)\right)}\right| \leqq b_{0}\left(\chi_{\varepsilon}\left(u ; r_{1}\right)-\chi_{\varepsilon}\left(u ; r_{1}^{\prime}\right)\right) .
$$


Taking into account (3.20), (3.21), and (3.25), we conclude from (3.15) that

$$
\left|\frac{d \psi_{\varepsilon}}{d u}\right| \leqq \frac{1}{\varepsilon}\left|\psi_{\varepsilon}\right|+b\left(\chi_{\varepsilon}\left(u ; r_{1}\right)-\chi_{\varepsilon}\left(u ; r_{1}^{\prime}\right)\right),
$$

where $b=b_{0} c+b_{1}$ and $c$ is the uniform bound of $y(u)$ in $\left[0, u_{0}\right.$ [. Integrating (3.26) yields:

$$
\left|\psi_{\varepsilon}\left(u_{1}\right)\right| \leqq e^{u_{1} / \varepsilon}\left\{\left|\psi_{\varepsilon}(0)\right|+b \int_{0}^{u_{1}}\left(\chi_{\varepsilon}\left(u ; r_{1}\right)-\chi_{\varepsilon}\left(u ; r_{1}^{\prime}\right) d u\right\} .\right.
$$

Now it holds:

$$
\begin{aligned}
\chi_{\varepsilon}\left(u ; r_{1}\right)-\chi_{\varepsilon}\left(u ; r_{1}^{\prime}\right) & =\left(r_{1}-r_{1}^{\prime}\right) \cdot \operatorname{mean}_{s \in\left[r_{1}^{\prime}, r_{1}\right]}\left\{\exp \left[\frac{1}{2} \int_{0}^{u_{1}}\left(\frac{\partial \bar{g}_{\varepsilon}}{\partial r}\right)_{\left(u^{\prime}, \chi_{\varepsilon}\left(u^{\prime}, s\right)\right)} d u^{\prime}\right]\right\} \\
& \leqq\left(r_{1}-r_{1}^{\prime}\right) \cdot e^{\left(u_{1}-u\right) / 2 \varepsilon} .
\end{aligned}
$$

Thus, denoting by $\eta_{\varepsilon}$ the modulus of continuity of $\partial h_{\varepsilon} / \partial r$ with respect to $r$ and by $\eta_{0}$ the modulus of continuity of the data $\partial h_{0} / \partial r$, we have

$$
\eta_{\varepsilon}\left(r_{1}-r_{1}^{\prime}\right) \leqq e^{u_{0} / \varepsilon}\left\{\eta_{0}\left(e^{u_{0} / 2 \varepsilon}\left(r_{1}-r_{1}^{\prime}\right)\right)+2 \varepsilon b\left(e^{u_{0} / 2 \varepsilon}-1\right)\left(r_{1}-r_{1}^{\prime}\right)\right\} .
$$

We conclude that $\partial h_{\varepsilon} / \partial r$ is uniformly continuous in $\left[0, u_{0}[\times[0, \infty[\right.$. The global existence of a classical solution follows. The facts that $h_{\varepsilon}=O\left(r^{-3}\right)$ and $\partial h_{\varepsilon} / \partial r$ $=O\left(r^{-4}\right)$ at each $u$ are easily deduced from a priori bounds. Finally, the uniqueness of the solution is proved by using the argument of Theorem 2 of [1].

\section{Section 4. Derivation of $\varepsilon$-Independent Estimates}

In this section we shall derive estimates for the solution of $\varepsilon$-regularized evolution equation in a region $r \geqq \delta, \delta$ any given positive real number, which are independent of $\varepsilon$ if $\varepsilon \leqq \delta / 2$.

At $u=0, h_{\varepsilon}$ coincides with the initial data of the original problem: $h_{\varepsilon}(0, r)$ $=h(0, r)=h_{0}(r)$. Before deriving the estimates, we shall for later reference compare at $u=0$ the quantities $g_{\varepsilon}$ and $\bar{g}_{\varepsilon}$ to the quantities $g$ and $\bar{g}$. In this paragraph all quantities are at $u=0$. Let

$$
A_{\varepsilon}=\int_{r}^{\infty}(h-\bar{h})^{2} \frac{d r}{r}, \quad A_{\varepsilon}=\int_{r}^{\infty}\left(h_{\varepsilon}-\overline{h_{\varepsilon}}\right)^{2} \frac{d r}{r+\varepsilon} .
$$

Then, since $h_{\varepsilon}=h$, and therefore $\bar{h}_{\varepsilon}=\frac{r}{r+\varepsilon} \bar{h}$, for $r \geqq \delta$ and $\varepsilon<\delta$, we have

$$
\begin{aligned}
\left|A-A_{\varepsilon}\right|= & \left|\int_{r}^{\infty}\left[\frac{1}{r}(h-\bar{h})^{2}-\frac{1}{r+\varepsilon}\left(h-\bar{h}+\frac{\varepsilon}{r+\varepsilon} \bar{h}\right)^{2}\right] d r\right| \leqq \varepsilon \int_{r}^{\infty} \frac{(h-\bar{h})^{2}}{r(r+\varepsilon)} d r \\
& +2 \varepsilon \int_{r}^{\infty} \frac{|h-\bar{h}| \bar{h} \mid}{(r+\varepsilon)^{2}} d r+\varepsilon^{2} \int_{r}^{\infty} \frac{\bar{h}^{2}}{(r+\varepsilon)^{3}} d r \leqq \frac{\varepsilon}{\delta} C(\delta),
\end{aligned}
$$

where

$$
C(\delta)=\int_{\delta}^{\infty}(|h-\bar{h}|+|\bar{h}|)^{2} \frac{d r}{r} .
$$


Hence for $r \geqq \delta$ it holds:

$$
e^{-4 \pi(\varepsilon / \delta) C(\delta)} \leqq \frac{g_{\varepsilon}}{g} \leqq e^{4 \pi(\varepsilon / \delta) C(\delta)}
$$

Let now $\varepsilon<\eta<\delta$. For $r \geqq \delta$, (4.3) implies that

$$
\bar{g}_{\varepsilon}(r) \geqq \frac{1}{r+\varepsilon} \int_{\eta}^{r} g_{\varepsilon}\left(r^{\prime}\right) d r^{\prime} \geqq \frac{1}{1+\varepsilon / \delta} e^{-4 \pi(\varepsilon / \eta) C(\eta)} \cdot \frac{1}{r} \int_{\eta}^{r} g\left(r^{\prime}\right) d r .
$$

Setting $r^{\prime}=s+\eta\left(1-\frac{s}{r}\right)$, we obtain

$$
\frac{1}{r} \int_{\eta}^{r} g\left(r^{\prime}\right) d r^{\prime}=\left(1-\frac{\eta}{r}\right) \cdot \frac{1}{r} \int_{0}^{r} g\left(s+\eta\left(1-\frac{s}{r}\right)\right) d s \leqq\left(1-\frac{\eta}{r}\right) \bar{g}(r),
$$

since $g(r)$ is a monotonically nondecreasing function of $r$. Thus for $r \geqq \delta$, we have

$$
\bar{g}_{\varepsilon} \geqq \frac{(1-\eta / \delta)}{(1+\varepsilon / \delta)} e^{-4 \pi(\varepsilon / \eta) C(\eta)} \bar{g} .
$$

For $r \geqq \delta,(4.3)$ implies that also:

$$
\bar{g}_{\varepsilon} \leqq \frac{1}{r+\varepsilon}\left(\int_{\eta}^{r} g_{\varepsilon} d r+\eta\right) \leqq\left(e^{4 \pi(\varepsilon / \eta) C(\eta)}+\frac{\eta}{\delta \bar{g}(\delta)}\right) \bar{g} .
$$

From (4.3) and (4.4) together with (4.5), we conclude that for each $\delta>0$ and for each $k>1$, there exists a $\varepsilon_{0}(\delta ; k)>0$ such that for all $\varepsilon \leqq \varepsilon_{0}(\delta ; k)$,

$$
\frac{1}{k} \frac{g}{\bar{g}} \leqq \frac{g_{\varepsilon}}{\bar{g}_{\varepsilon}} \leqq k \frac{g}{\bar{g}}
$$

holds for all $r \geqq \delta$. Also, $\varepsilon_{0}(\delta ; k)$ is a monotonically nondecreasing function of $\delta$. Consequently, for all $\varepsilon \leqq \varepsilon_{0}(\delta ; k)$, we have

$$
\frac{1}{k} \int_{\delta}^{r} \frac{g}{\bar{g}} d r \leqq \int_{\delta}^{r} \frac{g_{\varepsilon}}{\bar{g}_{\varepsilon}} d r \leqq k \int_{\delta}^{r} \frac{g}{\bar{g}} d r
$$

for all $r \geqq \delta$. It follows that

$$
\int_{\delta}^{r} \frac{g_{\varepsilon}}{\bar{g}_{\varepsilon}} d r \rightarrow \int_{\delta}^{r} \frac{g}{\bar{g}} d r
$$

and $m_{\varepsilon}(r) \rightarrow m(r)$ uniformly in $\left[\delta, r_{0}\right], r_{0}$ arbitrary. Since also

$$
M_{0, \varepsilon}-m_{\varepsilon}(r)=2 \pi \int_{r}^{\infty} \frac{\bar{g}_{\varepsilon}}{g_{\varepsilon}}\left(h_{\varepsilon}-\bar{h}_{\varepsilon}\right)^{2} d r \rightarrow 0
$$

for $r \rightarrow \infty$, uniformly in $\varepsilon\left(M_{0, \varepsilon}\right.$ stands for $\left.M_{\varepsilon}(0)\right)$, we conclude that $M_{0, \varepsilon} \rightarrow M_{0}$ as $\varepsilon \rightarrow 0$. Hence excluding the case of trivial initial data, $M_{0, \varepsilon} / M_{0} \rightarrow 1$ as $\varepsilon \rightarrow 0$. That is, for every $k>1$ there is a $\mu(k)>0$ such that for all $\varepsilon \leqq \mu(k)$ we have

$$
\frac{1}{k} M_{0} \leqq M_{0, \varepsilon} \leqq k M_{0}
$$


We shall find it convenient to assume $\delta \leqq \delta_{0}$, where

$$
\delta_{0}:=\min \left\{2 \mu(2), M_{0}\right\} .
$$

Then for all $\varepsilon \leqq \delta / 2$ we shall have $M_{0} / 2 \leqq M_{0, \varepsilon} \leqq 2 M_{0}$ and $2 M_{0, \varepsilon} \geqq \delta$. The above results will be referred to in Sect. 5 .

Lemma 2. For every $\varepsilon>0$, the following inequality holds:

$$
\int_{0}^{\infty} h_{\varepsilon}^{2}(u, r) d r \leqq \frac{u}{8 \pi}+\int_{0}^{\infty} h_{0}^{2}(r) d r .
$$

Proof. We have

$$
\begin{aligned}
\frac{\partial}{\partial u}\left(\int_{0}^{\infty} h_{\varepsilon}^{2} d r\right) & =\int_{0}^{\infty}\left[D_{\varepsilon}\left(h_{\varepsilon}^{2}\right)+\frac{1}{2} \bar{g}_{\varepsilon} \frac{\partial}{\partial r}\left(h_{\varepsilon}^{2}\right)\right] d r=\int_{0}^{\infty}\left[\frac{\partial \bar{g}_{\varepsilon}}{\partial r} h_{\varepsilon}\left(h_{\varepsilon}-\bar{h}_{\varepsilon}\right)-\frac{1}{2} \frac{\partial \bar{g}_{\varepsilon}}{\partial r} h_{\varepsilon}^{2}\right] d r \\
& =\int_{0}^{\infty} \frac{\left(g_{\varepsilon}-\bar{g}_{\varepsilon}\right)}{(r+\varepsilon)}\left[\frac{1}{2}\left(h_{\varepsilon}-\bar{h}_{\varepsilon}\right)^{2}-\frac{1}{2} \bar{h}_{\varepsilon}^{2}\right] d r .
\end{aligned}
$$

Therefore

$$
\frac{\partial}{\partial u}\left(\int_{0}^{\infty} h_{\varepsilon}^{2} d r\right) \leqq \frac{1}{2} \int_{0}^{\infty} g_{\varepsilon} \frac{\left(h_{\varepsilon}-\overline{h_{\varepsilon}}\right)^{2}}{(r+\varepsilon)} d r=\frac{1}{8 \pi} \int_{0}^{\infty} \frac{\partial g_{\varepsilon}}{\partial r} d r=\frac{1}{8 \pi}\left(1-g_{\varepsilon}(u, 0)\right) \leqq \frac{1}{8 \pi}
$$

Integrating this with respect to $u$ yields the lemma.

Lemma 3. For each $0<\delta \leqq \delta_{0}$ and each $\varepsilon \leqq \delta / 2$, the following estimate holds:

$$
\sup _{r \geqq \delta}\left|\bar{h}_{\varepsilon}(u, r)\right| \leqq b(u ; \delta) \text {, }
$$

where

$$
b(u ; \delta):=\frac{1}{\delta^{1 / 2}}\left(\frac{u}{8 \pi}+\int_{0}^{\infty} h_{0}^{2}(r) d r\right)^{1 / 2}
$$

Proof. We have

$$
\left|\bar{h}_{\varepsilon}(u, r)\right| \leqq \frac{1}{r} \int_{0}^{r}\left|h_{\varepsilon}\left(u, r^{\prime}\right)\right| d r^{\prime} \leqq \frac{1}{r^{1 / 2}}\left(\int_{0}^{r} h_{\varepsilon}^{2}\left(u, r^{\prime}\right) d r^{\prime}\right)^{1 / 2},
$$

hence

$$
\sup _{r \geqq \delta}\left|\bar{h}_{\varepsilon}(u, r)\right| \leqq \frac{1}{\delta^{1 / 2}}\left(\int_{0}^{\infty} h_{\varepsilon}^{2}(u, r) d r\right)^{1 / 2} .
$$

The conclusion then follows from Lemma 2.

Lemm 4. For each $0<\delta \leqq \delta_{0}$ and each $\varepsilon \leqq \delta / 2$, the following estimates hold:

$$
\sup _{r \geqq \delta}\left|h_{\varepsilon}(u, r)\right| \leqq c(u ; \delta) \quad \text { and } \sup _{r \geqq \delta}\left|\frac{\partial h_{\varepsilon}}{\partial r}(u, r)\right| \leqq c^{\prime}(u ; \delta) \text {. }
$$

Here

$$
c(u ; \delta):=e^{u / 2 \delta}\left(d_{0}+\frac{u}{2 \delta} b(u ; \delta)\right)
$$


and

$$
c^{\prime}(u ; \delta)=e^{u / \delta}\left\{d_{1}+\frac{u}{2 \delta^{2}}\left[3+4 \pi(b(u ; \delta)+c(u ; \delta))^{2}\right](b(u ; \delta)+c(u ; \delta))\right\},
$$

where

$$
d_{0}:=\sup _{r \geqq 0}\left|h_{0}(r)\right|, \quad d_{1}:=\sup _{r \geqq 0}\left|\frac{\partial h_{0}}{\partial r}(r)\right| .
$$

Proof. We integrate the $\varepsilon$-regularized evolution equation (2.7) along the $\varepsilon$-characteristic $\chi_{\varepsilon}\left(u ; r_{1}\right)$ through $r=r_{1}$ at $u=u_{1}$ :

$h_{\varepsilon}\left(u_{1}, r_{1}\right)=h\left(0, r_{0, \varepsilon}\right)+\int_{0}^{u_{1}}\left[\frac{1}{2(r+\varepsilon)}\left(g_{\varepsilon}-\bar{g}_{\varepsilon}\right) h_{\varepsilon}\right]_{\chi_{\varepsilon}} d u-\int_{0}^{u_{1}}\left[\frac{1}{2(r+\varepsilon)}\left(g_{\varepsilon}-\bar{g}_{\varepsilon}\right) \bar{h}_{\varepsilon}\right]_{\chi_{\varepsilon}} d u$.

Taking $r_{1} \geqq \delta$, along $\chi_{\varepsilon}$, we have

$$
\frac{1}{r+\varepsilon}\left(g_{\varepsilon}-\bar{g}_{\varepsilon}\right) \leqq \frac{1}{r_{1}+\varepsilon} \leqq \frac{1}{\delta} .
$$

Therefore, by Lemma 3,

$$
\int_{0}^{u_{1}}\left[\frac{1}{2(r+\varepsilon)}\left(g_{\varepsilon}-\bar{g}_{\varepsilon}\right)\left|\bar{h}_{\varepsilon}\right|\right]_{\chi_{\varepsilon}} d u \leqq \frac{1}{2 \delta} \int_{0}^{u_{1}} b(u ; \delta) d u .
$$

Thus setting

$$
x(u):=\sup _{r \geqq \delta}\left|h_{\varepsilon}(u ; r)\right|,
$$

we obtain from (4.6),

$$
x\left(u_{1}\right) \leqq d_{0}+\frac{1}{2 \delta} \int_{0}^{u_{1}} x(u) d u+\frac{1}{2 \delta} \int_{0}^{u_{1}} b(u ; \delta) d u .
$$

It follows that

$$
\sup _{r \geqq \delta}\left|h_{\varepsilon}(u, r)\right| \leqq c(u ; \delta) .
$$

We now integrate the derivative equation (3.7) along the $\varepsilon$-characteristic $\chi_{\varepsilon}$ :

$$
\begin{aligned}
\frac{\partial h_{\varepsilon}}{\partial r}\left(u_{1}, r_{1}\right)= & \frac{\partial h}{\partial r}\left(0, r_{0, \varepsilon}\right)+\int_{0}^{u_{1}}\left[\frac{\left(g_{\varepsilon}-\bar{g}_{\varepsilon}\right)}{(r+\varepsilon)} \frac{\partial h_{\varepsilon}}{\partial r}\right]_{\chi_{\varepsilon}} d u \\
& \left.+\int_{0}^{u_{1}}\left\{\frac{1}{2(r+\varepsilon)^{2}}\left[-3\left(g_{\varepsilon}-\bar{g}_{\varepsilon}\right)+4 \pi g_{\varepsilon}\left(h_{\varepsilon}-\bar{h}_{\varepsilon}\right)^{2}\right]\left(h_{\varepsilon}-\bar{h}_{\varepsilon}\right)\right]_{\chi_{\varepsilon}}\right\} d u .
\end{aligned}
$$

Using (4.7) and Lemma 3, we can estimate the second integral on the right by

$$
\frac{1}{2 \delta^{2}} \int_{0}^{u_{1}}\left[3+4 \pi(b(u ; \delta)+c(u ; \delta))^{2}\right](b(u ; \delta)+c(u ; \delta)) d u .
$$

Thus setting

$$
y(u):=\sup _{r \geqq \delta}\left|\frac{\partial h_{\varepsilon}}{\partial r}(u, r)\right| .
$$


We obtain from (4.8),

$$
y(u) \leqq d_{1}+\frac{1}{\delta} \int_{0}^{u_{1}} y(u) d u+\frac{1}{2 \delta^{2}} \int_{0}^{u_{1}}\left[3+4 \pi(b(u ; \delta)+c(u, \delta))^{2}\right](b(u ; \delta)+c(u ; \delta)) d u .
$$

It follows that

$$
\sup _{r \geqq \delta}\left|\frac{\partial h_{\varepsilon}}{\partial r}(u, r)\right| \leqq c^{\prime}(u ; \delta) .
$$

Lemma 5. The solution of the $\varepsilon$-regularized evolution equation has at each $u$ the following falloff property:

$$
\sup _{r \geqq 4 M_{0, \varepsilon}}\left\{\left(\frac{r}{4 M_{0, \varepsilon}}\right)^{3}\left|h_{\varepsilon}(u, r)\right|\right\} \leqq k_{\varepsilon}(u),
$$

and

$$
\sup _{r \geqq 4 M_{0, \varepsilon}}\left\{\left(\frac{r}{4 M_{0, \varepsilon}}\right)^{4}\left|4 M_{0, \varepsilon} \frac{\partial h_{\varepsilon}}{\partial r}(u, r)\right|\right\} \leqq k_{\varepsilon}^{\prime}(u),
$$

where $k_{\varepsilon}(u) \rightarrow k(u)$ and $k_{\varepsilon}^{\prime}(u) \rightarrow k^{\prime}(u)$ as $\varepsilon \rightarrow 0$, uniformly in $u$. Here:

$$
k(u):=e^{3 u / 32 M_{0}}\left\{\sup _{r \geqq 4 M_{0}}\left[\left(\frac{r}{4 M_{0}}\right)^{3}\left|h_{0}(r)\right|\right]+\frac{1}{8 \pi^{1 / 2}} \cdot \frac{u}{4 M_{0}}\right\},
$$

and

$$
\begin{aligned}
k^{\prime}(u):= & e^{u / 4 M_{0}}\left\{\sup _{r \geqq 4 M_{0}}\left[\left(\frac{r}{4 M_{0}}\right)^{4}\left|4 M_{0} \frac{\partial h_{0}}{\partial r}(r)\right|\right]+\left(\frac{1}{2 \pi^{1 / 2}}+\frac{3}{2} k(u)\right)\right. \\
& \left.\times\left[\frac{3}{4}+2 \pi\left(\frac{1}{2 \pi^{1 / 2}}+\frac{3}{2} k(u)\right)^{2}\right] \cdot \frac{u}{4 M_{0}}\right\} .
\end{aligned}
$$

Proof. For any differentiable function $f$ such that $f$ and $r \partial f / \partial r$ belong to $L^{2}(0, \infty)$ and $\lim _{r \rightarrow \infty} r f^{2}(r)=0$, it holds:

$$
r_{1} f^{2}\left(r_{1}\right) \leqq \int_{r_{1}}^{\infty} r^{2}\left(\frac{\partial f}{\partial r}\right)^{2} d r
$$

Let us take $f=\bar{h}_{\varepsilon}$ and $r_{1}=4 M_{0, \varepsilon}$ (by Lemma $1 \bar{h}_{\varepsilon}=O\left(r^{-1}\right)$, we then obtain:

$$
4 M_{0, \varepsilon} \bar{h}_{\varepsilon}^{2}\left(4 M_{0, \varepsilon}\right) \leqq\left|\int_{4 M_{0, \varepsilon}}^{\infty}(r+\varepsilon)^{2}\left(\frac{\partial \bar{h}_{\varepsilon}}{\partial r}\right)^{2}\right| d r \leqq \frac{M_{0, \varepsilon}}{\pi}
$$

(see $(2.10)$ ) since $\bar{g}_{\varepsilon} / g_{\varepsilon} \geqq 1 / 2$ for $r \geqq 4 M_{0, \varepsilon}$. Hence

$$
\left|\bar{h}_{\varepsilon}\left(4 M_{0, \varepsilon}\right)\right| \leqq \frac{1}{2 \pi^{1 / 2}} .
$$

For $r \geqq 4 M_{0, \varepsilon}$, we have

$$
\bar{h}_{\varepsilon}(r)=\frac{1}{r+\varepsilon}\left[\left(4 M_{0, \varepsilon}+\varepsilon\right) \bar{h}_{\varepsilon}\left(4 M_{0, \varepsilon}\right)+\int_{4 M_{0, \varepsilon}}^{r} h_{\varepsilon}\left(r^{\prime}\right) d r^{\prime}\right] .
$$


Taking into account (4.13) and setting

$$
x(u):=\sup _{r \geqq 4 M_{0, \varepsilon}}\left\{\left(\frac{r}{4 M_{0, \varepsilon}}\right)^{3}\left|h_{\varepsilon}(u, r)\right|\right\}
$$

(by Lemma $1, h_{\varepsilon}=O\left(r^{-3}\right)$ ) we may estimate $\bar{h}_{\varepsilon}$ for $r \geqq 4 M_{0, \varepsilon}$ by

$$
\begin{aligned}
\left|\bar{h}_{\varepsilon}(r)\right| & \leqq \frac{1}{r+\varepsilon}\left[\frac{\left(4 M_{0, \varepsilon}+\varepsilon\right)}{2 \pi^{1 / 2}}+\int_{4 M_{0, \varepsilon}}^{r} x \cdot\left(\frac{4 M_{0, \varepsilon}}{r^{\prime}}\right)^{3} d r^{\prime}\right] \\
& \leqq\left[\left(1+\frac{\varepsilon}{4 M_{0, \varepsilon}}\right) \cdot \frac{1}{2 \pi^{1 / 2}}+\frac{1}{2} x\right]\left(\frac{4 M_{0, \varepsilon}}{r}\right) .
\end{aligned}
$$

We also have

$$
\frac{g_{\varepsilon}-\bar{g}_{\varepsilon}}{2(r+\varepsilon)}=\frac{m_{\varepsilon}}{(r+\varepsilon)^{2}} g_{\varepsilon} \leqq \frac{M_{0, \varepsilon}}{r^{2}}
$$

Taking $r_{1} \geqq 4 M_{0, \varepsilon}$ and taking into account (4.15) and (4.16) we derive from (4.6) a linear integral inequality for $x(u)$ which implies

$$
x(u) \leqq k_{\varepsilon}(u)
$$

where

$$
\begin{aligned}
k_{\varepsilon}(u):= & e^{3 u / 32 M_{0, \varepsilon}}\left\{\sup _{r \geqq 4 M_{0, \varepsilon}}\left[\left(\frac{r}{4 M_{0, \varepsilon}}\right)^{3}\left|h_{0}(r)\right|\right]\right. \\
& \left.+\frac{1}{8 \pi^{1 / 2}}\left(1+\frac{\varepsilon}{4 M_{0, \varepsilon}}\right) \cdot \frac{u}{4 M_{0, \varepsilon}}\right\} .
\end{aligned}
$$

This establishes (4.9). We then obtain (4.10) by deriving from (4.8) a linear integral inequality for the quantity

$$
y(u):=\sup _{r \geqq 4 M_{0, \varepsilon}}\left\{\left(\frac{r}{4 M_{0, \varepsilon}}\right)^{4}\left|4 M_{0, \varepsilon} \frac{\partial h_{\varepsilon}}{\partial r}(u, r)\right|\right\},
$$

using the previous results.

Let us now denote $Q_{\delta}=\left\{(u, r) \mid 0 \leqq u \leqq u_{0}, r \geqq \delta\right\}$, where $0 \leqq \delta<\delta_{0}$ and $u_{0}$ is an arbitrary positive number.

Lemma 6. The family of functions $\left\{\partial h_{\varepsilon} / \partial r \mid 0<\varepsilon \leqq \delta / 2\right\}$ is equicontinuous in $Q_{\delta}$.

Proof. By Lemmas 3 and 4, the families of functions $\left\{h_{\varepsilon}-\bar{h}_{\varepsilon}\right\}$ and $\left\{\partial h_{\varepsilon} / \partial r\right\}$ are equibounded in $Q_{\delta}$ :

$$
\left|h_{\varepsilon}-\bar{h}_{\varepsilon}\right| \leqq C_{0} \text { and }\left|\partial h_{\varepsilon} / \partial r\right| \leqq C_{1},
$$

where $C_{0}$ and $C_{1}$ are independent of $\varepsilon$. It then follows from (3.7) that in $Q_{\delta}$,

$$
\left|D_{\varepsilon}\left(\frac{\partial h_{\varepsilon}}{\partial r}\right)\right| \leqq \frac{C_{1}}{\delta}+\frac{1}{2 \delta^{2}}\left(3+4 \pi C_{0}^{2}\right) C_{0}:=C_{2},
$$

and $C_{2}$ is independent of $\varepsilon$. As a consequence of this, we need only demonstrate the equicontinuity of the family $\left\{\partial h_{\varepsilon} / \partial r\right\}$ in $Q_{\delta}$ with respect to $r$. Equicontinuity with 
respect to $u$ would then follow, since we would have: (see (3.13))

$$
\begin{aligned}
& \left|\frac{\partial h_{\varepsilon}}{\partial r}\left(u_{1}, r_{1}\right)-\frac{\partial h_{\varepsilon}}{\partial r}\left(u_{1}-\Delta u_{1}, r_{1}\right)\right| \\
& \quad \leqq \int_{u_{1}-\Delta u_{1}}^{u_{1}} \mid\left(D_{\varepsilon}\left(\partial h_{\varepsilon} / \partial r\right)|d u+| \frac{\partial h_{\varepsilon}}{\partial r}\left(u_{1}-\Delta u_{1}, r_{1}+\Delta r_{1, \varepsilon}\right)-\frac{\partial h_{\varepsilon}}{\partial r}\left(u_{1}-\Delta u_{1}, r_{1}\right) \mid\right. \\
& \quad \leqq C_{2} \cdot \Delta u_{1}+\eta\left(\Delta r_{1, \varepsilon}\right) \leqq C_{2} \cdot \Delta u_{1}+\eta\left(\frac{1}{2} \Delta u_{1}\right)
\end{aligned}
$$

where $\eta$ denote the common modulus of continuity of the family $\left\{\partial h_{\varepsilon} / \partial r\right\}$ with respect to $r$.

Let now $\delta \leqq r_{1}^{\prime}<r_{1}$. Then, taking $u_{1} \leqq u_{0}$, the $\varepsilon$-characteristics $\chi_{\varepsilon, u_{1}}\left(u ; r_{1}^{\prime}\right)$ and $\chi_{\varepsilon, u_{1}}\left(u ; r_{1}\right)$ are contained in $Q_{\delta}$ for $0 \leqq u \leqq u_{1}$. Defining $\psi_{\varepsilon}(u)$ as in (3.14), we obtain from (3.15) and (3.21):

$$
\begin{aligned}
\frac{d \psi_{\varepsilon}}{d u}= & \left(\frac{\partial \bar{g}_{\varepsilon}}{\partial r}\right)_{\left(u, \chi_{\varepsilon}\left(u ; r_{1}\right)\right)} \cdot \psi_{\varepsilon}(u)+\left[\left(\frac{\partial \bar{g}_{\varepsilon}}{\partial r}\right)_{\left(u, \chi_{\varepsilon}\left(u ; r_{1}\right)\right)}-\left(\frac{\partial \bar{g}_{\varepsilon}}{\partial r}\right)_{\left(u, \chi_{\varepsilon}\left(u ; r_{1}^{\prime}\right)\right)}\right] \\
& \times\left(\frac{\partial h_{\varepsilon}}{\partial r}\right)_{\left(u, \chi_{\varepsilon}\left(u ; r_{1}^{\prime}\right)\right)}+f_{\varepsilon}\left(u, \chi_{\varepsilon}\left(u ; r_{1}\right)\right)-f_{\varepsilon}\left(u, \chi_{\varepsilon}\left(u ; r_{1}^{\prime}\right)\right),
\end{aligned}
$$

where $f_{\varepsilon}$ is given by (3.16). Using now (4.19), we can estimate in $Q_{\delta}$ (see (3.22) and (3.17)):

$$
\left|\frac{\partial^{2} \bar{g}_{\varepsilon}}{\partial r^{2}}\right| \leqq \frac{1}{\delta^{2}}\left(4 \pi C_{0}^{2}+2\right):=K_{0}
$$

and

$$
\left|\frac{\partial f_{\varepsilon}}{\partial r}\right| \leqq \frac{1}{2 \delta^{2}} C_{1}\left(3+12 \pi C_{0}^{2}\right)+\frac{2}{\delta^{3}} C_{0}\left(3+8 \pi C_{0}^{2}+4 \pi^{2} C_{0}^{4}\right):=K_{1},
$$

and $K_{0}$ and $K_{1}$ are independent of $\varepsilon$. It follows that

$$
\left|\left(\frac{\partial \bar{g}_{\varepsilon}}{\partial r}\right)_{\left(u, \chi_{\varepsilon}\left(u ; r_{1}\right)\right)}-\left(\frac{\partial \bar{g}_{\varepsilon}}{\partial r}\right)_{\left(u, \chi_{\varepsilon}\left(u ; r_{1}^{\prime}\right)\right)}\right| \leqq K_{0}\left(\chi_{\varepsilon}\left(u ; r_{1}\right)-\chi_{\varepsilon}\left(u ; r_{1}^{\prime}\right)\right),
$$

and

$$
\left|f_{\varepsilon}\left(u, \chi_{\varepsilon}\left(u ; r_{1}\right)\right)-f_{\varepsilon}\left(u, \chi_{\varepsilon}\left(u ; r_{1}^{\prime}\right)\right)\right| \leqq K_{1}\left(\chi_{\varepsilon}\left(u ; r_{1}\right)-\chi_{\varepsilon}\left(u ; r_{1}^{\prime}\right)\right) \text {. }
$$

Taking into account that (4.26), (4.25), (4.19) and the fact that in $Q_{\varepsilon}, \partial \bar{g}_{\varepsilon} / \partial r \leqq 1 / \delta$, we conclude from (4.22) that

$$
\left|\frac{d \psi_{\varepsilon}}{d u}\right| \leqq \frac{1}{\delta}\left|\psi_{\varepsilon}\right|+K\left(\chi_{\varepsilon}\left(u ; r_{1}\right)-\chi_{\varepsilon}\left(u ; r_{1}^{\prime}\right)\right)
$$

where $K=K_{0} C_{1}+K_{1}$. Integrating (4.27) yields

$$
\left|\psi_{\varepsilon}\left(u_{1}\right)\right| \leqq e^{u_{1} / \delta}\left\{\left|\psi_{\varepsilon}(0)\right|+K \int_{0}^{u_{1}}\left(\chi_{\varepsilon}\left(u ; r_{1}\right)-\chi_{\varepsilon}\left(u ; r_{1}^{\prime}\right)\right) d u\right\} .
$$


Now, since both $\varepsilon$-characteristics are contained in $Q_{\delta}$, it holds:

$$
\begin{aligned}
& \chi_{\varepsilon}\left(u ; r_{1}\right)-\chi_{\varepsilon}\left(u ; r_{1}^{\prime}\right)=\left(r_{1}-r_{1}^{\prime}\right) \cdot \underset{s \in\left[r_{1}^{\prime}, r_{1}\right]}{\operatorname{mean} \text { value }} \\
& \quad \times\left\{\exp \left[\frac{1}{2} \int_{u}^{u_{1}}\left(\frac{\partial \bar{g}_{\varepsilon}}{\partial r}\right)_{\left(u^{\prime}, \chi_{\varepsilon}\left(u^{\prime} ; s\right)\right)} d u^{\prime}\right]\right\} \leqq\left(r_{1}-r_{1}^{\prime}\right) e^{\left(u_{1}-u\right) / 2 \delta} .
\end{aligned}
$$

Denoting then by $\eta$ the modulus of continuity of $\partial h_{\varepsilon} / \partial r$ with respect to $r$ in $Q_{\delta}$ and by $\eta_{0}$ the modulus of continuity of the data $\partial h_{0} / \partial r$, we have

$$
\eta\left(r_{1}-r_{1}^{\prime}\right) \leqq e^{u_{0} / \delta}\left\{\eta_{0}\left(e^{u_{0} / 2 \delta}\left(r_{1}-r_{1}^{\prime}\right)\right)+2 \delta K\left(e^{u_{0} / 2 \delta}-1\right)\left(r_{1}-r_{1}^{\prime}\right)\right\} .
$$

Thus, since $K$ is independent of $\varepsilon$, the lemma follows.

Lemma 7. The family of functions $\left\{\bar{h}_{\varepsilon} \mid 0<\varepsilon \leqq \delta / 2\right\}$ is equicontinuous in $Q_{\varepsilon}$.

Proof. The equicontinuity of the family $\left\{\bar{h}_{\varepsilon}\right\}$ with respect to $r$ in $Q_{\delta}$ follows from the fact that the family $\left\{\partial \bar{h}_{\varepsilon} / \partial r=\left(h_{\varepsilon}-\bar{h}_{\varepsilon}\right) /(r+\varepsilon)\right\}$ is equibounded (by $\left.C_{0} / \delta\right)$ in $Q_{\delta}$. Thus we need only show equicontinuity with respect to $u$. Let again $r_{1}+\Delta r_{1, \varepsilon}$ denote the value of $r$ at which the $\varepsilon$-characteristic $\chi_{\varepsilon, u_{1}}\left(u ; r_{1}\right)$ through $\left(u_{1}, r_{1}\right)$ intersects the line $u=u_{1}-\Delta u_{1}, \Delta u_{1}>0$. We have:

$$
\begin{aligned}
& \left|\bar{h}_{\varepsilon}\left(u_{1}, r_{1}\right)-\bar{h}_{\varepsilon}\left(u_{1}-\Delta u_{1}, r_{1}\right) \leqq \int_{u_{1}-\Delta u_{1}}^{u_{1}}\left[D_{\varepsilon} \bar{h}_{\varepsilon}\right]_{\chi_{\varepsilon, u_{1}}}\left(u_{1} ; r_{1}\right) d u\right| \\
& \quad+\left|\bar{h}_{\varepsilon}\left(u_{1}-\Delta u_{1}, r_{1}+\Delta r_{1, \varepsilon}\right)-\bar{h}_{\varepsilon}\left(u_{1}-\Delta u_{1}, r_{1}\right)\right| .
\end{aligned}
$$

Since $\Delta r_{1, \varepsilon} \leqq \Delta u_{1} / 2,(4.19)$ implies that the second term on the right in (4.31) is bounded by

$$
\left|\bar{h}_{\varepsilon}\left(u_{1}-\Delta u_{1}, r_{1}+\Delta r_{1, \varepsilon}\right)-\bar{h}_{\varepsilon}\left(u_{1}-\Delta u_{1}, r_{1}\right)\right| \leqq \frac{C_{0}}{\delta} \Delta r_{1, \varepsilon} \leqq \frac{C_{0}}{2 \delta} \Delta u_{1} .
$$

By (2.12) and the $\varepsilon$-mass-flux relation (2.19), we can estimate the first term on the right in (4.31) as follows:

$$
\begin{aligned}
& \left|\int_{u_{1}-\Delta u_{1}}^{u_{1}}\left[D_{\varepsilon} \bar{h}_{\varepsilon}\right]_{\chi_{\varepsilon, u_{1}}\left(u ; r_{1}\right)} d u\right|=\left|\int_{u_{1}-\Delta u_{1}}^{u_{1}}\left[\frac{\xi_{\varepsilon}}{2(r+\varepsilon)}\right]_{\chi_{\varepsilon, u_{1}}\left(u ; r_{1}\right)} \mathrm{du}\right| \\
& \quad \leqq \frac{1}{2 \delta}\left(\int_{u_{1}-\Delta u_{1}}^{u_{1}}\left[\xi_{\varepsilon}^{2}\right]_{\chi_{\varepsilon, u_{1}}\left(u ; r_{1}\right)} d u\right)^{1 / 2}\left(\Delta u_{1}\right)^{1 / 2} \leqq \frac{1}{2 \delta}\left(\frac{M_{0, \varepsilon}}{\pi}\right)^{1 / 2}\left(\Delta u_{1}\right)^{1 / 2} .
\end{aligned}
$$

Hence we obtain

$$
\left|\bar{h}_{\varepsilon}\left(u_{1}, r_{1}\right)-\bar{h}_{\varepsilon}\left(u_{1}-\Delta u_{1}, r_{1}\right)\right| \leqq \frac{1}{2 \delta}\left[C_{0} \cdot \Delta u_{1}+\left(\frac{M_{0, \varepsilon}}{\pi}\right)^{1 / 2} \cdot\left(\Delta u_{1}\right)^{12}\right] .
$$

Thus, since $M_{0, \varepsilon} / M_{0} \rightarrow 1$ as $\varepsilon \rightarrow 0$, the lemma follows.

\section{Section 5. Compactness Arguments; Proof of Theorem 1}

We now begin the proof of Theorem 1 . We confine our attention to a fixed but arbitrary interval $0 \leqq u \leqq u_{0}$. In this section, $Q$ shall denote the domain 
$Q:=\left\{(u, r) \mid 0 \leqq u \leqq u_{0}, r>0\right\}, Q_{\delta}$ the domain $Q_{\delta}:=\{(u, r) \in Q \mid r \geqq \delta\}$, and $Q_{\delta, r_{0}}$ the domain $Q_{\delta, r_{0}}:=\left\{(u, r) \in Q \mid \delta \leqq r \leqq r_{0}\right\}$.

Let us set $\delta_{m}:=\delta_{0} / 2^{m}, m=0,1,2, \ldots$, and $\varepsilon_{n}:=\delta_{0} / 2^{n}, n=1,2,3, \ldots$.

We consider the sequence $\left\{h_{\varepsilon_{n}} \mid n=1,2,3, \ldots\right\}$ of solutions of the $\varepsilon_{n}$-regularized evolution equation taking the given initial data $h_{0}$ at $u=0$. By Lemma 4 the sequences $\left\{h_{\varepsilon_{n}}\right\},\left\{\partial h_{\varepsilon_{n}} / \partial r\right\}$ and (by Lemma 3 and the regularized evolution equation) also $\left\{\partial h_{\varepsilon_{n}} / \partial u\right\}$ are equibounded in $Q_{\delta_{m}}$ for each $m$ and each $n \geqq m+1$. We start with $m=0$; by the Ascoli-Arzela theorem we can select $a$ a subsequence $\left\{h_{\varepsilon_{n, 0}}\right\}$ converging uniformly on compact subsets $Q_{\delta_{0}, r_{0}}$ of $Q_{\delta_{0}}$ to $h_{\delta_{0}}$, a continuous function on $Q_{\delta_{0}}$. At the next step $m=1$, we can select a subsequence $\left\{h_{\varepsilon_{n, 1}}\right\}$ of the subsequence $\left\{h_{\varepsilon_{n, 0}}\right\}$ converging to $\left.h\right|_{\delta_{1}}$, a continuous function on $Q_{\delta_{1}}$. Now $\left.h\right|_{\delta_{1}}$ agrees with $\left.h\right|_{\delta_{0}}$ on $Q_{\delta_{0}}$, therefore $\left.h\right|_{\delta_{1}}$ extends $\left.h\right|_{\delta_{0}}$ to $Q_{\delta_{1}}$. We keep extracting subsequences in this way, that is extracting each subsequence $\left\{h_{\varepsilon_{n, m}}\right\}$ out of the previous subsequence $\left\{h_{\varepsilon_{n, m-1}}\right\}$; for each $m,\left.h\right|_{\delta_{m}}$ extends $\left.h\right|_{\delta_{m-1}}$ to $Q_{\delta_{m}}$.

Then, the diagonal subsequence whose $i^{\text {th }}$ term is the $i^{\text {th }}$ term of the subsequence $\left\{h_{\varepsilon_{n, 1-1}}\right\}$, converges uniformly on compact subsets $Q_{\delta, r_{0}}$ of $Q$ to $h$, a continuous function on $Q$ which for each $m$ agrees on $Q_{\delta_{m}}$ with $\left.h\right|_{\delta_{m}}$. We shall denote this diagonal subsequence simply by $\left\{h_{\varepsilon_{n}}\right\}$, keeping in mind that $n$ now ranges only over a certain subsequence of the sequence of positive integers. Lemma 4 implies that $h$ satisfies in each $Q_{\delta}$ the bound

$$
\sup _{r>\delta}|h(u, r)| \leqq c(u ; \delta),
$$

and Lemma 5 implies that at each $u, h=O\left(r^{-3}\right)$ for $r \rightarrow \infty$ and

$$
\sup _{r \geqq 4 M_{0}}\left\{\left(\frac{r}{4 M_{0}}\right)^{3}|h(u, r)|\right\} \leqq k(u) .
$$

By Lemma 6 the sequence $\left\{\partial h_{\varepsilon_{n}} / \partial r \mid n=1,2,3, \ldots\right\}$ is equicontinuous in $Q_{\delta_{m}}$ for each $m$ and each $n \geqq m+1$. Consequently, by the Ascoli-Arzela theorem, for each $m$ the subsequence $\left\{h_{\varepsilon_{n, m}}\right\}$ can be chosen so that the corresponding subsequence $\left\{\partial h_{\varepsilon_{n, m}} / \partial r\right\}$ converges uniformly on compact subsets $Q_{\delta_{m}, r_{0}}$ of $Q_{\delta_{m}}$ to $\left.h^{\prime}\right|_{\delta_{m}}$, a continuous function on $Q_{\delta_{m}}$. Then the diagonal subsequence will be such that the corresponding subsequence $\left\{\partial h_{\varepsilon_{n}} / \partial r\right\}$ converges uniformly on compact subsets $Q_{\delta, r_{0}}$ of $Q$ to $h^{\prime}$ a continuous function on $Q$. It follows that for each $r^{\prime}, r^{\prime \prime}>0$,

$$
\int_{r^{\prime \prime}}^{r^{\prime}} h^{\prime} d r=h\left(r^{\prime}\right)-h\left(r^{\prime \prime}\right),
$$

and therefore $h^{\prime}=\partial h / \partial r$ and $h$ is continuously differentiable with respect to $r$ in $Q$. Then Lemma 4 implies that $\partial h / \partial r$ satisfies in each $Q_{\delta}$ the bound

$$
\sup _{r \geqq \delta}\left|\frac{\partial h}{\partial r}(u, r)\right| \leqq c^{\prime}(u ; \delta),
$$

and Lemma 5 implies that at each $u, \partial h / \partial r=O\left(r^{-4}\right)$ for $r \rightarrow \infty$ and

$$
\sup _{r \geqq 4 M_{0}}\left\{\left(\frac{r}{4 M_{0}}\right)^{4}\left|4 M_{0} \frac{\partial h}{\partial r}(u, r)\right|\right\} \leqq k^{\prime}(u) .
$$


According to Lemma 2,

$$
\int_{0}^{\infty} h_{\varepsilon_{n}}^{2}(u, r) d r \leqq \frac{u}{8 \pi}+\int_{0}^{\infty} h_{0}^{2}(r) d r .
$$

Now for every $\delta>0$ and every $r_{0}>\delta$,

$$
\int_{\delta}^{r_{0}} h_{\varepsilon_{n}}^{2} d r \rightarrow \int_{\delta}^{r_{0}} h^{2} d r
$$

uniformly in $u$. Consider the sequence $\left\{\mathrm{f}_{m}\right\}$ of functions

$$
f_{m}:=\left\{\begin{array}{lll}
0 & \text { for } \quad r<\delta_{m} \text { and for } \quad r>m \\
h^{2} & \text { for } \quad \delta_{m} \leqq r \leqq m
\end{array} .\right.
$$

The $f_{m}$ form an increasing sequence of measurable functions such that

$$
\int_{0}^{\infty} f_{m} d r=\int_{\delta_{m}}^{m} h^{2} d r \leqq \frac{u}{8 \pi}+\int_{0}^{\infty} h_{0}^{2} d r
$$

By the monotone convergence theorem, $h^{2}$, which is the pointwise limit of $f_{m}$ for $m \rightarrow \infty$, is integrable on $(0, \infty)$. Thus (since $h$ is measurable, being continuous for $r>0) h \in L^{2}(0, \infty)$ at each $u$ and

$$
\int_{0}^{\infty} h^{2} d r \leqq \frac{u}{8 \pi}+\int_{0}^{\infty} h_{0}^{2} d r
$$

Let

$$
\zeta_{\varepsilon_{n}}:=\left(r+\varepsilon_{n}\right) \bar{h}_{\varepsilon_{n}}=\int_{0}^{r} h_{\varepsilon_{n}} d r
$$

By Lemma 3 and Lemma 7, the sequence $\left\{\bar{h}_{\varepsilon_{n}} \mid n=1,2,3 \ldots\right\}$ is equibounded and equicontinuous in $Q_{\delta_{m}}$ for each $m$ and $n \geqq m+1$. Hence the same is true for the sequence $\left\{\zeta_{\varepsilon_{n}} \mid n=1,2,3 \ldots\right\}$. Consequently, by the Ascoli-Arzela theorem, for each $m$ the subsequence $\left\{h_{\varepsilon_{n, m}}\right\}$ can be chosen so that the corresponding subsequence $\left\{\zeta_{\varepsilon_{n, m}}\right\}$ converges uniformly on compact subsets of $Q_{\delta_{m, r_{0}}}$ to $\zeta_{\delta_{m}}$, a continuous function on $Q_{\delta_{m}}$. Then the diagonal subsequence will be such that the corresponding subsequence $\left\{\zeta_{\varepsilon_{n}}\right\}$ converges uniformly on compact subsets $Q_{\delta, r_{0}}$ of $Q$ to $\zeta$, a continuous function on $Q$. Lemma 3 implies that for each $\delta>0$,

$$
\zeta(u, \delta) \leqq \delta b(u ; \delta) .
$$

It follows that at each $u, \zeta(\delta) \rightarrow 0$ as $\delta \rightarrow 0$. On the other hand, we have $\delta \zeta_{\varepsilon_{n}} / \partial r$ $=h_{\varepsilon_{n}} \rightarrow h$, uniformly on compact subsets of $Q$. Hence

$$
\zeta_{\varepsilon_{n}}(r)-\zeta_{\varepsilon_{n}}(\delta)=\int_{\delta}^{r}\left(\partial \zeta_{\varepsilon_{n}} / \partial r\right) d r \rightarrow \int_{\delta}^{r} h d r
$$

But $\zeta_{\varepsilon_{n}}(r)-\zeta_{\varepsilon_{n}}(\delta) \rightarrow \zeta(r)-\zeta(\delta)$. Therefore $\zeta(r)-\zeta(\delta)=\int_{\delta}^{r} h d r$. Since, by the previous paragraph, $h \in L^{1}\left(0, r_{0}\right), r_{0}$ arbitrary, letting $\delta \rightarrow 0$, we obtain

$$
\zeta(r)=\int_{0}^{r} h d r=r \bar{h} .
$$


We conclude that $\bar{h}_{\varepsilon_{n}}$ converges to $\bar{h}$ uniformly on compact subsets $Q_{\delta, r_{0}}$ of $Q$. Also, $\bar{h}$ is a continuous function on $Q$ and satisfies in each $Q_{\delta}$ the bound

$$
\sup _{r \geqq \delta}|\bar{h}(u, r)| \leqq b(u ; \delta) .
$$

Furthermore, the fact that, at each $u, h$ belongs to $L^{2}(0, \infty)$ implies that at each $u, \bar{h}$ belongs also to $L^{2}(0, \infty)$ and $\delta \bar{h}^{2}(u, \delta) \rightarrow 0$ as $\delta \rightarrow 0$. In addition, (4.13) implies that

$$
\left|\bar{h}\left(u, 4 M_{0}\right)\right| \leqq \frac{1}{2 \pi^{1 / 2}},
$$

and (5.2) implies that

$$
\sup _{r \geqq 4 M_{0}}\left\{\left(\frac{r}{4 M_{0}}\right)|\bar{h}(u, r)|\right\}
$$

is bounded by a continuous function of $u$.

The facts that $\bar{k}_{\varepsilon_{n}}, h_{\varepsilon_{n}}, \partial h_{\varepsilon_{n}} / \partial r$ converge uniformly in each $Q_{\delta, r_{0}}$ to $\bar{h}, h, \partial h / \partial r$, respectively, and that $\bar{h}_{\varepsilon_{n}}, h_{\varepsilon_{n}}, \partial h_{\varepsilon_{n}} / \partial r$ tend to zero as $r \rightarrow \infty$ uniformly in $u$ and $n$, imply that $\bar{h}_{\varepsilon_{n}}, h_{\varepsilon_{n}}, \partial h_{\varepsilon_{n}} / \partial r$ converge uniformly in each $Q_{\delta}$ to $\bar{h}, h, \partial h / \partial r$, respectively.

For each $\delta>0$ and each $r_{0}>\delta$,

$$
\int_{r}^{r_{0}}\left(h_{\varepsilon_{n}}-\bar{h}_{\varepsilon_{n}}\right)^{2} \frac{d r}{r+\varepsilon_{n}} \rightarrow \int_{r}^{r_{0}}(h-\bar{h})^{2} \frac{d r}{r},
$$

uniformly in $r \in\left[\delta, r_{0}\right]$ and $u \in\left[0, u_{0}\right]$. By Lemma 5 (see (4.15)) since $M_{0} / 2 \leqq M_{0, \varepsilon_{n}}$ $\leqq 2 M_{0}$ for $r \geqq 8 M_{0}$, we have

$$
\left|h_{\varepsilon_{n}}(r)-\bar{h}_{\varepsilon_{n}}(r)\right| \leqq C \cdot\left(\frac{8 M_{0}}{r}\right)
$$

where $C$ is independent of $r$ and $n$. Hence, if $r_{0} \geqq 8 M_{0}$,

$$
\int_{r_{0}}^{\infty}\left(h_{\varepsilon_{n}}-\bar{h}_{\varepsilon_{n}}\right)^{2} \frac{d r}{r+\varepsilon_{n}} \leqq \int_{r_{0}}^{\infty} C^{2}\left(\frac{8 M_{0}}{r}\right)^{2} \frac{d r}{r}=\frac{C^{2}}{2}\left(\frac{8 M_{0}}{r_{0}}\right)^{2} .
$$

The same inequality must hold for $\int_{r_{0}}^{\infty}(h-\bar{h})^{2} \frac{d r}{r}$. Given now any $\eta>0$, we can choose $r_{0}$ such that $\frac{C^{2}}{r}\left(\frac{8 M_{0}}{r}\right)^{2}<\eta / 3$.

By (5.8), we can then choose $N_{1}$ such that for all $n \geqq N_{1}$,

$$
\left|\int_{r}^{r_{0}}(h-\bar{h})^{2} \frac{d r}{r}-\int_{r}^{r_{0}}\left(h_{\varepsilon_{n}}-\bar{h}_{\varepsilon_{n}}\right)^{2} \frac{d r}{r+\varepsilon_{n}}\right|<\eta / 3 .
$$

Then,

$$
\begin{aligned}
& \left|\int_{r}^{\infty}(h-\bar{h})^{2} \frac{d r}{r}-\int_{r}^{\infty}\left(h_{\varepsilon_{n}}-\bar{h}_{\varepsilon_{n}}\right)^{2} \frac{d r}{r+\varepsilon_{n}}\right| \leqq\left|\int_{r}^{r_{0}}(h-\bar{h})^{2} \frac{d r}{r}-\int_{r}^{r_{0}}\left(h_{\varepsilon_{n}}-\bar{h}_{\varepsilon_{n}}\right)^{2} \frac{d r}{r+\varepsilon_{n}}\right| \\
& \quad+\int_{r_{0}}^{\infty}(h-\bar{h})^{2} \frac{d r}{r}+\int_{r_{0}}^{\infty}\left(h_{\varepsilon_{n}}-\overline{h_{\varepsilon_{n}}}\right)^{2} \frac{d r}{r+\varepsilon_{n}} \leqq \frac{\eta}{3}+\frac{\eta}{3}+\frac{\eta}{3}=\eta .
\end{aligned}
$$


We conclude that

$$
A_{\varepsilon_{n}}:=\int_{r}^{\infty}\left(h_{\varepsilon_{n}}-\bar{h}_{\varepsilon_{n}}\right)^{2} \frac{d r}{r+\varepsilon_{n}} \rightarrow A:=\int_{r}^{\infty}(h-\bar{h})^{2} \frac{d r}{r},
$$

uniformly in $Q_{\delta}$ for any $\delta>0$. Therefore also

$$
g_{\varepsilon_{n}}:=\exp \left(-4 \pi A_{\varepsilon_{n}}\right) \rightarrow g:=\exp (-4 \pi A)
$$

uniformly in $Q_{\delta}$ for any $\delta>0$. The fact that, at each $u, h-\bar{h} \in L^{2}(0, \infty)$ implies that, at each $u, \delta A(u, \delta) \rightarrow 0$.

We know then that $g$ is a continuous function in $Q$. At each $u, g(u, r)$ is positive, continuous, and monotonically non-decreasing in $r$. Hence $g(u, r)$ tends to a limit as $r \rightarrow 0$. We set $g(u, 0):=\lim _{r \rightarrow 0} g(u, r)$. Then $g$ is continuous with respect to $r$ even at $r=0$. The sequence $\{g(u, 1 / m) \mid m=1,2,3, \ldots\}$ is a non-increasing sequence of continuous functions of $u$ which are $>0$ and $\leqq 1$. It follows that $g(u, 0)$ $=\lim g(u, 1 / m)$ is a measurable function of $u$ which is $\geqq 0$ and $\leqq 1$.

We now consider $\bar{g}:=\frac{1}{r} \int_{0}^{r} g d r$. It follows from the above that $\bar{g}$ is continuous with respect to $r$ for all $r \geqq 0$ and $\bar{g}(u, 0)=g(u, 0)$. We shall now show that $\bar{g}$ is also continuous with respect to $u$ in $Q$. Given any $\eta>0$ and $r_{0}>\eta / 2$, by the continuity of $g$ with respect to $u$ in $Q$ we can choose $\varepsilon$ such that for $\left|u-u^{\prime}\right|<\varepsilon$, we have

$$
\left|g(u, r)-g\left(u^{\prime}, r\right)\right| \leqq \eta / 2 r_{0} \text { for all } r \in\left[\eta / 2, r_{0}\right] .
$$

Then

$$
\begin{gathered}
\left|\int_{0}^{r} g(u, s) d s-\int_{0}^{r} g\left(u^{\prime}, s\right) d s\right| \leqq \int_{0}^{\eta / 2}\left|g(u, s)-g\left(u^{\prime}, s\right)\right| d s \\
\quad+\int_{\eta / 2}^{r}\left|g(u, s)-g\left(u^{\prime}, s\right)\right| d s<\frac{\eta}{2}+\left(\frac{\eta}{2 r_{0}}\right) \cdot r_{0}=\eta .
\end{gathered}
$$

Hence $\int_{0}^{r} g d r$, and therefore $\bar{g}$ is continuous with respect to $u$ in $Q$.

We know from the above that

$$
\int_{\gamma}^{\delta} g_{\varepsilon_{n}}(u, r) d r \rightarrow \int_{\gamma}^{\delta} g(u, r) d r,
$$

uniformly in $u$ for $\gamma, \delta$ fixed, $\delta \geqq \gamma>0$. Thus for every $\gamma>0$ and every $\eta>0$, there exists $N(\gamma, \eta)$ such that for all $u \in\left[0, u_{0}\right]$, we have

$$
\left|\int_{\gamma}^{\delta} g(u, r) d r-\int_{\gamma}^{\delta} g_{\varepsilon_{n}}(u, r) d r\right|<\eta, \text { for all } n \geqq N .
$$

Given now any $\eta^{\prime}>0$, let $N^{\prime}=N\left(\eta^{\prime} / 2, \eta^{\prime} / 2\right)$. Then we have

$$
\left|\int_{\eta^{\prime} / 2}^{\delta} g(u, r) d r-\int_{\eta^{\prime} / 2}^{\delta} g_{\varepsilon_{n}}(u, r) d r\right|<\eta^{\prime} / 2 \text { for all } n \geqq N^{\prime},
$$


and therefore

$$
\begin{aligned}
& \left|\int_{0}^{\delta} g(u, r) d r-\int_{0}^{\delta} g_{\varepsilon_{n}}(u, r) d r\right| \leqq\left|\int_{\eta^{\prime} / 2}^{\delta} g(u, r) d r-\int_{\eta^{\prime} / 2}^{\delta} g_{\varepsilon_{n}}(u, r) d r\right| \\
& \quad+\int_{0}^{\eta^{\prime}}\left|g(u, r)-g_{\varepsilon_{n}}(u, r)\right| d r<\frac{\eta^{\prime}}{2}+\frac{\eta^{\prime}}{2}=\eta^{\prime} \quad \text { for all } n \geqq N^{\prime} .
\end{aligned}
$$

We conclude that

$$
\int_{0}^{\delta} g_{\varepsilon_{n}}(u, r) d r
$$

converges uniformly in $u$ to $\int_{0}^{\delta} g(u, r) d r$. For $r \geqq \delta$, we write

$$
r \bar{g}(r)=\int_{0}^{\delta} g(u, s) d s+\int_{\delta}^{r} g(u, s) d s .
$$

Since

$$
\int_{\delta}^{r} g_{\varepsilon_{n}}(u, s) d s
$$

converges to $\int_{\delta}^{r} g(u, s) d s$ uniformly in $Q_{\delta, r_{0}}, r_{0}$ arbitrary, we conclude that

$$
\left(r+\varepsilon_{n}\right) \bar{g}_{\varepsilon_{n}}=\int_{0}^{\delta} g_{\varepsilon_{n}}(u, s) d s+\int_{\delta}^{r} g_{\varepsilon_{n}}(u, s) d s
$$

converges uniformly in $Q_{\delta, r_{0}}$ to $r \bar{g}$. Therefore $\bar{g}_{\varepsilon_{n}} \rightarrow \bar{g}$ uniformly in compact subsets $Q_{\delta, r_{0}}$ of $Q$. Furthermore, the fact that for $r \geqq r_{0}$ we have

$$
\bar{g}_{\varepsilon_{n}}(r)=\frac{\left(r_{0}+\varepsilon_{n}\right)}{\left(r+\varepsilon_{n}\right)} \bar{g}_{\varepsilon_{n}}\left(r_{0}\right)+\frac{1}{r+\varepsilon_{n}} \int_{r_{0}}^{r} g_{\varepsilon_{n}}\left(r^{\prime}\right) d r^{\prime},
$$

and

$$
\bar{g}(r)=\frac{r_{0}}{r} \bar{g}\left(r_{0}\right)+\frac{1}{r} \int_{r_{0}}^{r} g\left(r^{\prime}\right) d r^{\prime},
$$

together with the fact that $g_{\varepsilon_{n}} \rightarrow g$ uniformly in $Q_{\delta}$, implies that $\bar{g}_{\varepsilon_{n}} \rightarrow \bar{g}$ uniformly in $Q_{\delta}$, for any $\delta>0$.

Let us recall the function $\varepsilon_{0}(\delta ; k)$ which is defined in the first paragraph of Sect. 4. We may assume that $\varepsilon_{0}(\delta ; 2) \leqq \delta / 2$. Let $N(m)$ denote the smallest positive integer such that $\varepsilon_{N} \leqq \varepsilon_{0}\left(\delta_{m} ; 2\right)$.

We shall now show that at each $u, g / \bar{g} \in L^{1}\left(0, r_{1}\right), r_{1}$ arbitrary. First, for each $m$

$$
\int_{\delta_{m}}^{r_{1}}\left(\frac{g_{\varepsilon_{n}}}{\bar{g}_{\varepsilon_{n}}}\right)(u, r) d r \rightarrow \int_{\delta_{m}}^{r_{1}}\left(\frac{g}{\bar{g}}\right)(u, r) d r
$$

uniformly in $u$, since $\bar{g}_{\varepsilon_{n}}\left(u, \delta_{m}\right)$ is uniformly in $n$ and $u$ bounded from below by a positive constant. By the $\varepsilon$-integral identity (2.22), for each $u_{1} \geqq 0$, we have

$$
\int_{\delta_{m}}^{r_{1}}\left(\frac{g_{\varepsilon_{n}}}{\bar{g}_{\varepsilon_{n}}}\right)\left(u_{1}, r\right) d r \leqq \int_{\delta_{m}}^{r_{0, \varepsilon_{n}}}\left(\frac{g_{\varepsilon_{n}}}{\bar{g}_{\varepsilon_{n}}}\right)(0, r) d r .
$$


Since $r_{0, \varepsilon_{n}} \leqq r_{1}+u_{1} / 2$, if $n \geqq N(m)$ (see first paragraph of Sect. 4)

$$
\begin{aligned}
\int_{\delta_{m}}^{r_{0, \varepsilon_{n}}}\left(\frac{g_{\varepsilon_{n}}}{\bar{g}_{\varepsilon_{n}}}\right)(0, r) d r & \leqq \int_{\delta_{m}}^{r_{1}+u_{1} / 2}\left(\frac{g_{\varepsilon_{n}}}{\bar{g}_{\varepsilon_{n}}}\right)(0, r) d r \leqq 2 \int_{\delta_{m}}^{r_{1}+u_{1} / 2}\left(\frac{g}{\bar{g}}\right)(0, r) d r \\
& \leqq 2 \int_{0}^{r_{1}+u_{1} / 2}\left(\frac{g}{\bar{g}}\right)(0, r) d r:=C\left(r_{1}+u_{1} / 2\right)
\end{aligned}
$$

Hence for all $n \geqq N(m)$,

$$
\int_{\delta_{m}}^{r_{1}}\left(\frac{g_{\varepsilon_{n}}}{\bar{g}_{\varepsilon_{n}}}\right)(u, r) d r \leqq C,
$$

and $C$ is independent of either $n$ or $m$. It follows that also

$$
\int_{\delta_{m}}^{r_{1}}\left(\frac{g}{\bar{g}}\right)(u, r) d r \leqq C .
$$

Consider the functions

$$
f_{m}:=\left\{\begin{array}{lll}
g / \bar{g} & \text { for } & r \geqq \delta_{m} \\
0 & \text { for } & r<\delta_{m}
\end{array} .\right.
$$

At each $u$, the sequence $\left\{f_{m} \mid m=0,1,2, \ldots\right\}$ is an increasing sequence of functions in $L^{1}\left(0, r_{1}\right), r_{1}$ arbitrary, such that

$$
\int_{0}^{r_{1}} f_{m}(u, r) d r<C \quad \text { (independent of } m \text { ) }
$$

By the monotone convergence theorem $g / \bar{g}$, which is the pointwise limit of the $f_{m}$ for $m \rightarrow \infty$, belongs at each $u$ to $L^{1}\left(0, r_{1}\right), r_{1}$ arbitrary, and

$$
\int_{0}^{r_{1}}\left(\frac{g}{\bar{g}}\right)\left(u_{1}, r\right) d r \leqq C\left(r_{1}+u_{1} / 2\right) .
$$

We shall now show:

Proposition 1. The property that, at each $u, g / \bar{g} \in L^{1}\left(0, r_{1}\right), r_{1}$ arbitrary, together with the finiteness of $M(u)$, is equivalent to the property that, at each $u, h \in L^{2}(0, \infty)$.

Proof. Since

$$
\begin{aligned}
\int_{\delta}^{r_{1}} \frac{g}{\bar{g}} d r= & -\int_{\delta}^{r_{1}} r d \log \left(r_{1} / r \bar{g}\right)=-r_{1} \log \left(1 / \bar{g}\left(r_{1}\right)\right)+\delta \log \left(r_{1} / \delta \bar{g}(\delta)\right) \\
& +\int_{\delta}^{r_{1}} \log \left(r_{1} / r \bar{g}\right) d r,
\end{aligned}
$$

$g / \bar{g} \in L^{1}\left(0, r_{1}\right) \quad$ implies that $\log (1 / \bar{g}) \in L^{1}\left(0, r_{1}\right)$, and therefore that $\log (1 / g) \in L^{1}\left(0, r_{1}\right)$. On the other hand,

$$
\int_{0}^{r_{1}} \log (1 / g) d r=r_{1} \log \left(1 / g\left(r_{1}\right)\right)+4 \pi \int_{0}^{r_{1}}(h-\bar{h})^{2} d r,
$$

and if we take $r_{1}=4 M$, we have

$$
\int_{r_{1}}^{\infty}(h-\bar{h})^{2} d r \leqq M / \pi
$$


Thus the integrability of $\log (1 / g)$ on $\left(0, r_{1}\right), r_{1}$ arbitrary, together with the finiteness of $M$ implies that $h-\bar{h} \in L^{2}(0, \infty)$. Finally since

$$
\int_{\delta}^{\infty} h^{2} d r=\int_{\delta}^{\infty}(h-\bar{h})^{2} d r-\delta \bar{h}^{2}(\delta),
$$

the square integrability of $h-\bar{h}$ with respect to $r$ implies the square integrability of $h$ with respect to $r$. The reverse implication is established by considering that

$$
\int_{0}^{\infty} \log (1 / g) d r=4 \pi \int_{0}^{\infty} h^{2} d r
$$

and

$$
\int_{0}^{r_{1}}\left[\frac{g}{\bar{g}}-1-\log \left(\frac{g}{\bar{g}}\right)\right] d r=r_{1} \log \left(1 / \bar{g}\left(r_{1}\right)\right)+\int_{0}^{r_{1}} \log (1 / g) d r .
$$

For each $\left(u_{1}, r_{1}\right) \in Q$, we define the characteristic $\chi_{u_{1}}\left(u ; r_{1}\right)$ through $r=r_{1}$ at $u=u_{1}$ to be the solution of the ordinary differential equation $\frac{d \chi}{d u}=-\frac{1}{2} \bar{g}(u, \chi)$, satisfying the condition $\chi\left(u_{1}\right)=r_{1}$. The existence and uniqueness of $\chi_{u_{1}}\left(u ; r_{1}\right)$ for all $\left(u_{1}, r_{1}\right) \in Q$ is guaranteed by the continuity of $\bar{g}$ and $\partial \bar{g} / \partial r$ in $Q$. We shall now show that the $\varepsilon_{n}$-characteristics $\chi_{\varepsilon_{n}}$ converge uniformly to the characteristics $\chi$. The $\varepsilon_{n}$ characteristic $\chi_{\varepsilon_{n, u_{1}}}\left(u ; r_{1}\right)$ through $r=r_{1}$ at $u=u_{1}$ is the solution of the ordinary differential equation,

$$
\frac{d \chi_{\varepsilon_{n}}}{d u}=-\frac{1}{2} \bar{g}_{\varepsilon_{n}}\left(u, \chi_{\varepsilon_{n}}\right),
$$

satisfying the condition $\chi_{\varepsilon_{n}}\left(u_{1}\right)=r_{1}$. Thus, we have

$$
\frac{d\left(\chi_{\varepsilon_{n}}-\chi\right)}{d u}=-\frac{1}{2}\left[\bar{g}_{\varepsilon_{n}}\left(u, \chi_{\varepsilon_{n}}\right)-\bar{g}(u, \chi)\right],
$$

and $\left(\chi_{\varepsilon_{n}}-\chi\right)\left(u_{1}\right)=0$. Integrating, we obtain

$$
\begin{aligned}
\left(\chi_{\varepsilon_{n}}-\chi\right)(u)= & \frac{1}{2} \int_{u}^{u_{1}}\left[\bar{g}_{\varepsilon_{n}}\left(u^{\prime}, \chi_{\varepsilon_{n}}\left(u^{\prime}\right)\right)-\bar{g}\left(u^{\prime}, \chi\left(u^{\prime}\right)\right)\right] d u^{\prime} \\
= & \frac{1}{2} \int_{u}^{u_{1}}\left[\bar{g}_{\varepsilon_{n}}\left(u^{\prime}, \chi\left(u^{\prime}\right)\right)-\bar{g}\left(u^{\prime}, \chi\left(u^{\prime}\right)\right)\right] d u^{\prime} \\
& +\frac{1}{2} \int_{u}^{u_{1}}\left[\bar{g}_{\varepsilon_{n}}\left(u^{\prime}, \chi_{\varepsilon_{n}}\left(u^{\prime}\right)\right)-\bar{g}_{\varepsilon_{n}}\left(u^{\prime}, \chi\left(u^{\prime}\right)\right)\right] d u^{\prime} .
\end{aligned}
$$

We set $y_{\varepsilon_{n}}(u):=\left|\chi_{\varepsilon_{n}}(u)-\chi(u)\right|$. Then $y_{\varepsilon_{n}}\left(u_{1}\right)=0$. Since $\left(u_{1}, r_{1}\right) \in Q$, we have $\left(u_{1}, r_{1}\right) \in Q_{\delta}$ for some $\delta>0$, and therefore $\left(u, \chi_{\varepsilon_{n}}(u)\right) \in Q_{\delta}$ and $(u, \chi(u)) \in Q_{\delta}$ for all $u \leqq u_{1}$. By the uniform convergence of $\bar{g}_{\varepsilon_{n}}$ to $\bar{g}$ in $Q_{\delta}$ we have $\mid \bar{g}_{\varepsilon_{n}}\left(u^{\prime}, \chi(u)\right)$ $-\bar{g}\left(u^{\prime}, \chi\left(u^{\prime}\right)\right) \mid \leqq \eta_{n}$, where $\eta_{n} \rightarrow 0$ for $n \rightarrow \infty$. Also the fact that the sequence $\left\{\partial \bar{g}_{\varepsilon_{n}} / \partial r\right\}$ is equibounded in $Q_{\delta}$,

$$
\sup _{Q_{\delta}} \frac{\partial \bar{g}_{\varepsilon_{n}}}{\partial r} \leqq \frac{1}{\delta}
$$


implies that

$$
\left|\bar{g}_{\varepsilon_{n}}\left(u^{\prime}, \chi_{\varepsilon_{n}}\left(u^{\prime}\right)\right)-\bar{g}_{\varepsilon_{n}}\left(u^{\prime}, \chi\left(u^{\prime}\right)\right)\right| \leqq \frac{1}{\delta} \cdot y_{\varepsilon_{n}}\left(u^{\prime}\right)
$$

Hence we obtain from (5.11) the inequality

$$
y_{\varepsilon_{n}}(u) \leqq \frac{1}{2} \eta_{n} \cdot\left(u_{1}-u\right)+\frac{1}{2 \delta} \int_{u}^{u_{1}} y_{\varepsilon_{n}}\left(u^{\prime}\right) d u^{\prime}
$$

It follows that $y_{\varepsilon_{n}}(u) \leqq \eta_{n} \cdot \delta\left(e^{\left(u_{1}-u\right) / 2 \delta}-1\right)$. We conclude that $y_{\varepsilon_{n}} \rightarrow 0$ uniformly in $u$ for $n \rightarrow \infty$, and therefore the $\varepsilon_{n}$-characteristics $\chi_{\varepsilon_{n}}$ converge uniformly to the characteristics $\chi$.

The function $h_{\varepsilon_{n}}$ satisfies the integral equation

$$
h_{\varepsilon_{n}}\left(u_{1}, r_{1}\right)=h_{0}\left(\chi_{\varepsilon_{n}}\left(0 ; r_{1}\right)\right)+\int_{0}^{u_{1}}\left[\frac{\left(g_{\varepsilon_{n}}-\bar{g}_{\varepsilon_{n}}\right)}{2\left(r+\varepsilon_{n}\right)}\left(h_{\varepsilon_{n}}-\bar{h}_{\varepsilon_{n}}\right)\right]_{\left(u, \chi_{\varepsilon_{n}}\left(u ; r_{1}\right)\right)} d u .
$$

Let $\left(u_{1}, r_{1}\right) \in Q$. Then $\left(u_{1}, r_{1}\right) \in Q_{\delta}$ for some $\delta>0$, and we know that for each $\delta>0$,

$$
\begin{aligned}
& h_{\varepsilon_{n}} \rightarrow h \text { uniformly in } Q_{\delta}, \\
& \bar{h}_{\varepsilon_{n}} \rightarrow \bar{h} \text { uniformly in } Q_{\delta}, \\
& g_{\varepsilon_{n}} \rightarrow g \text { uniformly in } Q_{\delta}, \\
& \bar{g}_{\varepsilon_{n}} \rightarrow \bar{g} \text { uniformly in } Q_{\delta},
\end{aligned}
$$

and $h, \bar{h}, g, \bar{g}$ are all continuous functions in $Q$. We also know that $\chi_{\varepsilon_{n}}\left(u ; r_{1}\right)$ $\rightarrow \chi\left(u ; r_{1}\right)$ uniformly in $u$. It follows that for $n \rightarrow \infty$ :

and

$$
h_{\varepsilon_{n}}\left(u_{1}, r_{1}\right) \rightarrow h\left(u_{1}, r_{1}\right), \quad h_{0}\left(\chi_{\varepsilon_{n}}\left(0 ; r_{1}\right)\right) \rightarrow h_{0}\left(\chi\left(0 ; r_{1}\right)\right)
$$

$$
\int_{0}^{u_{1}}\left[\frac{\left(g_{\varepsilon_{n}}-\tilde{g}_{\varepsilon_{n}}\right)}{2\left(r+\varepsilon_{n}\right)}\left(h_{\varepsilon_{n}}-\bar{h}_{\varepsilon_{n}}\right)\right]_{\left(u, \chi_{\varepsilon_{n}}\left(u ; r_{1}\right)\right)} d u \rightarrow \int_{0}^{u_{1}}\left[\frac{(g-\bar{g})}{2 r}(h-\bar{h})\right]_{\left(u, \chi\left(u ; r_{1}\right)\right)} d u .
$$

Therefore, for all $\left(u_{1}, r_{1}\right) \in Q$ it holds:

$$
h\left(u_{1}, r_{1}\right)=h_{0}\left(\chi\left(0 ; r_{1}\right)\right)+\int_{0}^{u_{1}}\left[\frac{(g-\bar{g})}{2 r}(h-\bar{h})\right]_{\left(u, \chi\left(u ; r_{1}\right)\right)} d u .
$$

We conclude that $h$ satisfies in $Q$ the nonlinear evolution equation in the integral sense.

We shall now show that $h$ is continuously differentiable with respect to $u$ in $Q$. We know that $\partial h / \partial r$ is a continuous in $Q$. We set $f:=\frac{(g-\bar{g})}{2 r}(h-\bar{h})$. Given $\left(u_{1}, r_{1}\right) \in Q$ and $\Delta u_{1}$, let $r_{1}+\Delta r_{1}$ be the value of $r$ at which the characteristic $\chi$ through $\left(u_{1}, r_{1}\right)$ intersects the line $u=u_{1}-\Delta u_{1}$. Then from (5.12) we obtain

$$
\begin{aligned}
& \frac{\left(h\left(u_{1}, r_{1}\right)-h\left(u_{1}-\Delta u_{1}, r_{1}\right)\right)}{\Delta u_{1}} \\
& \quad=\frac{1}{\Delta u_{1}} \int_{u_{1}-\Delta u_{1}}^{u_{1}} f\left(u, \chi\left(u ; r_{1}\right)\right) d u+\frac{\left(h\left(u_{1}-\Delta u_{1}, r_{1}+\Delta r_{1}\right)-h\left(u_{1}-\Delta u_{1}, r_{1}\right)\right)}{\Delta r_{1}} \cdot \frac{\Delta r_{1}}{\Delta u_{1}},
\end{aligned}
$$


and we have

$$
\frac{\Delta r_{1}}{\Delta u_{1}}=\frac{1}{\Delta u_{1}} \int_{u_{1}-\Delta u_{1}}^{u_{1}} \frac{1}{2} \bar{g}\left(u, \chi\left(u ; r_{1}\right)\right) d u .
$$

Since $h$ is known to be continuously differentiable with respect to $r$ in $Q$, as $\Delta u_{1} \rightarrow 0$ the right side of (5.13) tends to the limit $\left(f+\frac{\partial h}{\partial r} \cdot \frac{1}{2} \bar{g}\right)\left(u_{1}, r_{1}\right)$. Hence $h$ is continuously differentiable with respect to $u$ in $Q$ and

$$
\frac{\partial h}{\partial u}=\frac{1}{2 r}(g-\bar{g})(h-\bar{h})+\frac{1}{2} \bar{g} \frac{\partial h}{\partial r} .
$$

We conclude that $h \in C^{1}(Q)$ and satisfies the nonlinear evolution equation in the differential sense in $Q$.

From the $\varepsilon$-integral identity (2.22) it follows that for $r_{0} \geqq \delta_{0}$ but otherwise arbitrary and for each $m$ and $n \geqq N(m)$ :

$$
\iint_{Q_{\delta_{m}, r_{0}}} \frac{g_{\varepsilon_{n}}}{\bar{g}_{\varepsilon_{n}}^{2}} \frac{\xi_{\varepsilon_{n}}^{2}}{\left(r+\varepsilon_{n}\right)} d u d r \leqq \frac{C\left(r_{0}+u_{0} / 2\right)}{2 \pi}
$$

(considering the fact that $Q_{\delta_{m}, r_{0}} \subset \overline{Q_{\delta_{m}, \varepsilon_{n}}\left(u_{0}, r_{0}\right)}$ and taking into account (5.10)). The constant $C$ depends on $r_{0}$ and $u_{0}$ but is independent of either $n$ or $m$. Thus the sequence

$$
\left\{\frac{g_{\varepsilon_{n}}^{1 / 2}}{\bar{g}_{\varepsilon_{n}}} \frac{\xi_{\varepsilon_{n}}}{\left(r+\varepsilon_{n}\right)} \mid n=1,2,3, \ldots\right\}
$$

is contained in the closed ball of radius $C / 2 \pi$ in $L^{2}\left(Q_{\delta_{m}, r_{0}}\right)$ for any $r_{0} \geqq \delta_{0}$ and each $m$ and $n \geqq N(m)$. In virtue of the weak compactness of the closed balls in $L^{2}$, we can choose for each $m$ the subsequence $\left\{h_{\varepsilon_{n, m}}\right\}$ so that the corresponding subsequence

$$
\left\{\frac{g_{\varepsilon_{n, m}}^{1 / 2}}{\bar{g}_{\varepsilon_{n, m}}} \frac{\xi_{\varepsilon_{n, m}}}{\left(r+\varepsilon_{n, m}\right)^{1 / 2}}\right\}
$$

converges weakly in $L^{2}\left(Q_{\delta_{m}, r_{0}}\right)$ for any $r_{0} \geqq \delta_{0}$ to a function defined on $Q_{\delta_{m}}$, which we denote by

$$
\left.\left(\frac{g^{1 / 2}}{\bar{g}} \frac{\xi}{r^{1 / 2}}\right)\right|_{\delta_{m}}
$$

and which belongs to the closed ball of radius $C / 2 \pi L^{2}\left(Q_{\delta_{m}, r_{0}}\right), r_{0}$ arbitrary. Then the diagonal subsequence will be such that the corresponding subsequence

$$
\left\{\frac{g_{\varepsilon_{n}}^{1 / 2}}{\bar{g}_{\varepsilon_{n}}} \frac{\xi_{\varepsilon_{n}}}{\left(r+\varepsilon_{n}\right)^{1 / 2}}\right\}
$$

converges weakly in $L^{2}$ on compact subsets $Q_{\delta, r_{0}}$ of $Q$ to

$$
\frac{g^{1 / 2}}{\bar{g}} \frac{\xi}{r^{1 / 2}}
$$


a function on $Q$ whose restriction to each $Q_{\delta_{m}}$ equals $\left.\left(g^{1 / 2} \xi / \bar{g} r^{1 / 2}\right)\right|_{\delta_{m}}$. Consider the measurable functions

$$
f_{m}:=\left\{\begin{array}{lll}
0 & \text { for } & (u, r) \notin Q_{\delta_{m}} \\
\left.\left(\frac{g^{1 / 2}}{\bar{g}} \frac{\xi}{r^{1 / 2}}\right)\right|_{\delta_{m}} & \text { for } & (u, r) \in Q_{\delta_{m}} .
\end{array}\right.
$$

Setting

$$
Q\left(r_{0}\right):=\left\{(u, r) \in Q \mid r \leqq r_{0}\right\},
$$

the sequence $\left\{g_{m}^{2} \mid m=0,1,2, \ldots\right\}$ is an increasing sequence of functions in $L^{1}\left(Q\left(r_{0}\right)\right), r_{0}$ arbitrary, such that

$$
\iint_{Q\left(r_{0}\right)} f_{m}^{2} d u d r \leqq C / 2 \pi
$$

The function $g^{1 / 2} \xi / \bar{g} r^{1 / 2}$, which is the pointwise limit of the $f_{m}$ for $m \rightarrow \infty$, is measurable, and by the monotone convergence theorem belongs to $L^{2}\left(Q\left(r_{0}\right)\right), r_{0}$ arbitrary, and

$$
\iint_{Q\left(r_{0}\right)} \frac{g}{\bar{g}^{2}} \frac{\xi^{2}}{r} d u d r \leqq C / 2 \pi
$$

By the above paragraph, for any $L^{2}$ function $\xi$ whose support is a compact set in $Q$ we have:

$$
\iint_{Q} \frac{g_{\varepsilon_{n}}^{1 / 2}}{\bar{g}_{\varepsilon_{n}}} \frac{\xi_{\varepsilon_{n}}}{\left(r+\varepsilon_{n}\right)^{1 / 2}} \zeta d u d r \rightarrow \iint_{Q} \frac{g^{1 / 2}}{\bar{g}} \frac{\zeta}{r^{1 / 2}} \zeta d u d r .
$$

Since $g_{\varepsilon_{n}}^{1 / 2} / \tilde{g}_{\varepsilon_{n}}$ is not less than 1 and converges uniformly in $Q_{\delta}$ for any $\delta>0$ to $g^{1 / 2} / \bar{g}$, (5.14) implies that for any $C^{\infty}$ function $\phi$ whose support is compact and contained in the interior of $Q$, we have

$$
\iint_{Q} \xi_{\varepsilon_{n}} \phi d u d r \rightarrow \iint_{Q} \xi \phi \mathrm{dudr} .
$$

Now

$$
\frac{\partial \xi_{\varepsilon_{n}}}{\partial r}=\bar{g}_{\varepsilon_{n}} \frac{\left(h_{\varepsilon_{n}}-\bar{h}_{\varepsilon_{n}}\right)}{\left(r+\varepsilon_{n}\right)}
$$

(see (2.13)). Hence, for any $C^{\infty}$ function $\phi$ whose support is compact and contained in the interior of $Q$ it holds:

$$
-\iint_{Q} \tilde{g}_{\varepsilon_{n}} \frac{\left(h_{\varepsilon_{n}}-\bar{h}_{\varepsilon_{n}}\right)}{\left(r+\varepsilon_{n}\right)} \phi d u d r=\iint_{Q} \xi_{\varepsilon_{n}} \frac{\partial \phi}{\partial r} d u d r .
$$

Since $\bar{g}_{\varepsilon_{n}}\left(h_{\varepsilon_{n}}-\bar{h}_{\varepsilon_{n}}\right) /\left(r+\varepsilon_{n}\right)$ converges uniformly in $Q_{\delta}$ for any $\delta>0$ to $\bar{g}(h-\bar{h}) / r$, the left-hand side of (5.16) converges to $-\iint_{Q} \bar{g} \frac{(h-\bar{h})}{r} \phi d u d r$. By (5.15) the right-hand side of (5.16) converges to $\iint_{Q} \xi \frac{\partial \phi}{\partial r} d u d r$. Therefore, for any $C^{\infty}$ function $\phi$ whose support is compact and contained in the interior of $Q$, we have

$$
-\iint_{Q} \bar{g} \frac{(h-\bar{h})}{r} \phi d u d r=\iint_{Q} \xi \frac{\partial \phi}{\partial r} d u d r .
$$


We conclude that $\xi$ is weakly differentiable with respect to $r$ in $Q$ and

$$
\frac{\partial \xi}{\partial r}=\bar{g} \frac{(h-\bar{h})}{r} .
$$

Since $g / \bar{g} \geqq 1$, the fact that $g^{1 / 2} \xi / \bar{g} r^{1 / 2} \in L^{2}\left(Q\left(r_{0}\right)\right)$ implies that $\xi / \bar{g}^{1 / 2} r^{1 / 2} \in L^{2}\left(Q\left(r_{0}\right)\right)$. Thus, for almost all $u \xi(u, r) / \bar{g}^{1 / 2}(u, r) r^{1 / 2} \in L^{2}\left(0, r_{0}\right)$. Let us set $r=r_{0} e^{-s}$ and $\xi^{*}(u, s)=\xi(u, r), \bar{g}^{*}(u, s)=\bar{g}(u, r)$. Then since,

$$
\int_{0}^{\infty} \frac{\xi^{* 2}}{\bar{g}^{*}}(u, s) d s=\int_{0}^{r_{0}} \frac{\xi^{2}}{\bar{g}}(u, r) \frac{d r}{r},
$$

$\left(\xi^{*} / \bar{g}^{* 1 / 2}\right)(u, \cdot) \in L^{2}(0, \infty)$ for almost all $u$. By $(5.17)$,

Therefore

$$
\frac{\partial \xi^{*}}{\partial s}=-r \frac{\partial \xi}{\partial r}=-\bar{g}(h-\bar{h}) \text {. }
$$

$$
\begin{aligned}
\int_{0}^{\infty} \frac{1}{\bar{g}^{*}}\left(\frac{\partial \xi^{*}}{\partial s}\right)^{2} d s & =\int_{0}^{r_{0}} \bar{g}(h-\bar{h})^{2} \frac{d r}{r} \leqq \int_{0}^{r_{0}} g(h-\bar{h})^{2} \frac{d r}{r} \\
& =\int_{0}^{r_{0}} \frac{1}{4 \pi} \frac{\partial g}{\partial r} d r=\frac{1}{4 \pi}\left(g\left(r_{0}\right)-g(0)\right) \leqq \frac{1}{4 \pi} .
\end{aligned}
$$

Since $\bar{g}^{*} \leqq 1$, we conclude that for almost all $u \xi^{*}(u, \cdot)$ belongs to the Sobolev space $H_{1}(0, \infty)$, and in fact than $\xi^{*} \in L^{2}\left(0, r_{0} ; H_{1}(0, \infty)\right)$. By the Sobolev imbedding theorem for almost all $u \xi^{*}(u, \cdot) \in C^{1 / 2}\left[0, \infty\left[\right.\right.$ and $\xi^{*}(u, s) \rightarrow 0$ as $s \rightarrow \infty$. It follows that for almost all $u, \xi(u, r)$ is continuous with respect to $r$ and $\xi(u, r) \rightarrow 0$ as $r \rightarrow 0$. Since

$$
\xi(r)=\xi(\delta)+\int_{\delta}^{r} \bar{g}(h-\bar{h}) \frac{d r}{r},
$$

letting $\delta \rightarrow 0$, we conclude that

$$
\xi(r)=\lim _{\delta \rightarrow 0} \int_{\delta}^{r} \bar{g}(h-\bar{h}) \frac{d r}{r} .
$$

We note that the function $\bar{g}(h-\bar{h}) / r$ is not necessarily Lebesgue integrable on $\left(0, r_{0}\right)$. However for almost all $u$ the above limit exists and equals $\xi(r)$. The fact that $\xi / \bar{g}^{1 / 2} r^{1 / 2} \in L^{2}\left(Q\left(r_{0}\right)\right)$ implies of course that $\left(\xi / \bar{g}^{1 / 2}\right)(\cdot, \delta) \in L^{2}\left(0, u_{0}\right)$ for some $\delta>0$. Then, as a consequence of $(5.20) \xi / \bar{g}^{1 / 2}(\cdot, r) \in L^{2}\left(0, u_{0}\right)$ for all $r>0$. It follows also that the restriction of $\xi$ to the characteristic $\chi_{u_{1}}\left(\cdot, r_{1}\right)$ through each $\left(u_{1}, r_{1}\right) \in Q$ belongs to $L^{2}\left(0, u_{1}\right)$. For every $s_{0} \in[0, \infty[$, we have the Sobolev inequality

$$
\sup _{s \geqq s_{0}}\left\{\xi^{* 2}(s)\right\} \leqq c \int_{s_{0}}^{\infty}\left(\xi^{* 2}+\left(\frac{\partial \xi^{*}}{\partial s}\right)^{2}\right) d s,
$$

where $c$ is a universal constant, independent of $s_{0}$. Since $\bar{g}^{*}$ is a monotonically nonincreasing function of $s,(5.22)$ implies that

$$
\frac{\xi^{* 2}\left(s_{0}\right)}{\bar{g}^{*}\left(s_{0}\right)} \leqq \frac{c}{\bar{g}^{*}\left(s_{0}\right)} \int_{s_{0}}^{\infty}\left(\xi^{* 2}+\left(\frac{\partial \xi^{*}}{\partial s}\right)^{2}\right) d s \leqq c \int_{s_{0}}^{\infty} \frac{1}{\bar{g}^{*}}\left(\xi^{* 2}+\left(\frac{\partial \xi^{*}}{\partial s}\right)^{2}\right) d s
$$


Hence

$$
\sup _{s \in[0, \infty[}\left\{\left(\frac{\xi^{* 2}}{\bar{g}^{*}}\right)(s)\right\} \leqq c \int_{0}^{\infty} \frac{1}{\bar{g}^{*}}\left(\xi^{* 2}+\left(\frac{\partial \xi^{*}}{\partial s}\right)^{2}\right) d s .
$$

Taking into account (5.18) and (5.19), we obtain from (5.23) that

$$
\sup _{\left.r \in] 0, r_{0}\right]}\left\{\left(\frac{\xi^{2}}{\bar{g}}\right)(r)\right\} \leqq c\left\{\int_{0}^{r_{0}} \frac{\xi^{2}}{\bar{g}} \frac{d r}{r}+\frac{1}{4 \pi}\left(g\left(r_{0}\right)-g(0)\right)\right\} \text {. }
$$

Since $g$ is continuous with respect to $r$ at $r=0,(5.24)$ implies, if we let $r_{0} \rightarrow 0$, that at almost all $u \xi^{2} / \bar{g} \rightarrow 0$ as $r \rightarrow 0$. Integrating now (5.24) with respect to $u$ we obtain

$$
\int_{0}^{u_{0}} \sup _{r \in\left[0, r_{0}\right]}\left\{\left(\frac{\xi^{2}}{\bar{g}}\right)(u, r)\right\} d u \leqq c\left\{\iint_{Q\left(r_{0}\right)} \frac{\xi^{2}}{\bar{g}} \frac{d r}{r} d u+\frac{1}{4 \pi} \int_{0}^{u_{0}}\left(g\left(u, r_{0}\right)-g(u, 0)\right) d u\right\} .
$$

Since

$$
\xi(r)=\xi\left(r_{0}\right)+\int_{r_{0}}^{r} \bar{g}(h-\bar{h}) \frac{d r}{r}
$$

we have

$$
\left(\frac{\xi^{2}}{\bar{g}}\right)(r) \leqq 2\left(\frac{\xi^{2}}{\bar{g}}\right)\left(r_{0}\right)+\frac{2}{\bar{g}}\left(\int_{r_{0}}^{r} \bar{g}(h-\bar{h}) \frac{d r}{r}\right)^{2} .
$$

It follows that $r \geqq r_{0}\left(r_{0}>0\right),\left(\xi^{2} / \bar{g}\right)(r) \leqq 2\left(\xi^{2} / \bar{g}\right)\left(r_{0}\right)+C$, where $C$ is a continuous function of $u$ (considering the falloff property of $h-\bar{h}$ for $r \rightarrow \infty$ ). We then conclude from (5.25) that

$$
\sup _{r \geqq 0}\left\{\frac{\xi}{\bar{g}^{1 / 2}}(u, r)\right\} \in L^{2}\left(0, u_{0}\right) .
$$

Since, by dominated convergence,

$$
\int_{0}^{u_{0}}(g(u, r)-g(u, 0)) d u \rightarrow 0 \quad \text { as } \quad r \rightarrow 0,
$$

letting $r_{0} \rightarrow 0$ in (5.25), we obtain

$$
\int_{0}^{u_{0}}\left(\frac{\xi^{2}}{\bar{g}}\right)(u, r) d u \rightarrow 0 \quad \text { as } \quad r \rightarrow 0 .
$$

We summarize the results of the last paragraph in:

Proposition 2. At almost all $u, \xi / \bar{g}^{1 / 2}$ is a continuous and uniformly bounded function of $r$ such that $\xi / \bar{g}^{1 / 2} \rightarrow 0$ as $r \rightarrow 0$. Also, at each $r \xi / \bar{g}^{1 / 2} \in L^{2}\left(0, u_{0}\right), u_{0}$ arbitrary. Furthermore,

$$
\sup _{r \geqq 0}\left|\frac{\xi}{\bar{g}^{1 / 2}}(u, r)\right| \in L^{2}\left(0, u_{0}\right)
$$

and

$$
\int_{0}^{u_{0}}\left(\frac{\xi^{2}}{\bar{g}}\right)(u, r) d u \rightarrow 0 \quad \text { as } \quad r \rightarrow 0 .
$$


According to (2.12)

$$
\frac{\partial \bar{h}_{\varepsilon_{n}}}{\partial u}=\frac{1}{2} \bar{g}_{\varepsilon_{n}} \frac{\left(h_{\varepsilon_{n}}-\bar{h}_{\varepsilon_{n}}\right)}{\left(r+\varepsilon_{n}\right)}+\frac{\xi_{\varepsilon_{n}}}{2\left(r+\varepsilon_{n}\right)} .
$$

For any $C^{\infty}$ function $\phi$ whose support is compact and contained in the interior of $Q$, we then have

$$
\iint_{Q}\left(\frac{1}{2} \bar{g}_{\varepsilon_{n}} \frac{\left(h_{\varepsilon_{n}}-\bar{h}_{\varepsilon_{m}}\right)}{\left(r+\varepsilon_{n}\right)}+\frac{\xi_{\varepsilon_{n}}}{2\left(r+\varepsilon_{n}\right)}\right) \phi \mathrm{dudr}=-\iint_{Q} \bar{h}_{\varepsilon_{n}} \frac{\partial \phi}{\partial u} d u d r .
$$

Since $\bar{h}_{\varepsilon_{n}}$ converges to $\bar{h}$ uniformly in $Q_{\delta}$ for any $\delta>0$, the right-hand side of (5.26) converges to $-\iint_{Q} \bar{h} \frac{\partial \phi}{\partial u} d u d r$. Since also $h_{\varepsilon_{n}}$ and $\bar{g}_{\varepsilon_{n}}$ converge to $h$ and $\bar{g}$ respectively, uniformly in $Q_{\delta}$ for any $\delta>0$,

$$
\iint_{Q} \frac{1}{2} \bar{g}_{\varepsilon_{n}} \frac{\left(h_{\varepsilon_{n}}-\bar{h}_{\varepsilon_{n}}\right)}{\left(r+\varepsilon_{n}\right)} \phi d u d r \rightarrow \iint_{Q}^{1} \frac{1}{2} \bar{g} \frac{(h-\bar{h})}{r} \phi d u d r .
$$

Now, $\phi / r$ is also a $C^{\infty}$ function whose support is compact and contained in the interior of $Q$; consequently, by $(5.15)$

$$
\iint_{Q} \xi_{\varepsilon_{n}} \frac{\phi}{\left(r+\varepsilon_{n}\right)} d u d r \rightarrow \iint_{Q} \xi \frac{\phi}{r} d u d r .
$$

We conclude that

$$
-\iint_{Q} \bar{h} \frac{\partial \phi}{\partial u} d u d r=\iint_{Q}\left(\frac{1}{2} g \frac{(h-\bar{h})}{r}+\frac{\xi}{2 r}\right) \phi d u d r .
$$

Hence $\bar{h}$ is weakly differentiable with respect to $u$ in $Q$ and $D \bar{h}=\frac{\xi}{2 r}$. Consider now $A:=\int_{r}^{\infty}(h-\bar{h})^{2} \frac{d r}{r}$. It follows from the preceding that $A$ is weakly differentiable with respect to $u$ in $Q$ and

$$
\begin{aligned}
D A & =\frac{1}{2 r} \bar{g}(h-\bar{h})^{2}+\int_{r}^{\infty} 2(h-\bar{h})\left[D h+\frac{1}{2} \bar{g} \frac{\partial h}{\partial r}-\frac{1}{2} \bar{g} \frac{\partial \bar{h}}{\partial r}-D \bar{h}\right] \frac{d r}{r} \\
& =\frac{1}{2 r} \bar{g}(h-\bar{h})^{2}+\int_{r}^{\infty}(h-\bar{h})\left[\frac{\partial \bar{g}}{\partial r}(h-\bar{h})+\bar{g} \frac{\partial h}{\partial r}-\frac{\bar{g}}{r}(h-\bar{h})-\frac{\xi}{r}\right] \frac{d r}{r} .
\end{aligned}
$$

Taking into account the fact that

$$
\frac{1}{r} \frac{\partial \bar{g}}{\partial r}(h-\bar{h})^{2}+\bar{g} \frac{(h-\bar{h})}{r} \frac{\partial h}{\partial r}-\frac{\bar{g}}{r^{2}}(h-\bar{h})^{2}=\frac{g}{2 r^{2}}(h-\bar{h})^{2}+\frac{\partial}{\partial r}\left[\bar{g} \frac{(h-\bar{h})^{2}}{2 r}\right],
$$

we obtain

$$
D A=\int_{r}^{\infty}\left[\frac{1}{2} g(h-\bar{h})-\xi\right](h-\bar{h}) \frac{d r}{r^{2}} .
$$

Since $g=e^{-4 \pi A}, g$ is weakly differentiable with respect to $u$ in $A$ and $D g=-4 \pi g D A$. 
We shall now compute the evolution law of $\frac{1}{r} \int_{\delta}^{r} g d r, \delta>0$. We have

$$
\begin{aligned}
D\left(\frac{1}{r} \int_{\delta}^{r} g d r\right) & =\frac{1}{2} \frac{\bar{g}}{r^{2}}\left(\int_{\delta}^{r} g d r\right)-\frac{1}{2 r} \bar{g} g+\frac{1}{r} \int_{\delta}^{r}\left(D g+\frac{1}{2} \bar{g} \frac{\partial g}{\partial r}\right) d r \\
& =\frac{1}{2} \frac{\bar{g}}{r^{2}}(r \bar{g}-\delta \bar{g}(\delta))-\frac{1}{2 r} \bar{g} g-\frac{4 \pi}{r} \int_{\delta}^{r}\left(g D A+\frac{1}{2} \bar{g} g \frac{\partial A}{\partial r}\right) d r
\end{aligned}
$$

We can write

$$
\int_{\delta}^{r} g D A d r=\int_{\delta}^{r} D A d(r \bar{g})=r \bar{g} D A-\delta \bar{g}(\delta) D A(\delta)-\int_{\delta}^{r} r \bar{g} \frac{\partial D A}{\partial r} d r .
$$

Substituting this in (5.28), we obtain

$$
\begin{aligned}
D\left(\frac{1}{r} \int_{\delta}^{r} g d r\right)= & -\frac{1}{2} \frac{\bar{g}}{r^{2}} \delta \bar{g}(\delta)+\frac{4 \pi}{r} \delta \bar{g}(\delta) D A(\delta)-\frac{1}{2 r} \bar{g}(g-\bar{g})-4 \pi \bar{g} D A \\
& +\frac{4 \pi}{r} \int_{\delta}^{r} \bar{g}\left(r \frac{\partial D A}{\partial r}-\frac{1}{2} g \frac{\partial A}{\partial r}\right) d r .
\end{aligned}
$$

From (5.27), we have

$$
r \frac{\partial D A}{\partial r}-\frac{1}{2} g \frac{\partial A}{\partial r}=\frac{(h-\bar{h})}{r} \xi
$$

Hence (see 5.17).

$$
\int_{\delta}^{r} \bar{g}\left(r \frac{\partial D A}{\partial r}-\frac{1}{2} g \frac{\partial A}{\partial r}\right) d r=\int_{\delta}^{r} \bar{g} \frac{(h-\bar{h})}{r} \xi d r=\int_{\delta}^{r} \xi \frac{\partial \xi}{\partial r} d r=\frac{1}{2} \xi^{2}-\frac{1}{2} \xi^{2}(\delta) .
$$

Substituting (5.30) in (5.29), we conclude that

$$
D\left(\frac{1}{r} \int_{\delta}^{r} g d r\right)=f_{\delta}-\frac{1}{2 r} \bar{g}(g-\bar{g})-4 \pi \bar{g} D A+\frac{2 \pi}{r} \xi^{2},
$$

where

$$
f_{\delta}:=-\frac{1}{2} \frac{\bar{g}}{r^{2}} \delta \bar{g}(\delta)-\frac{2 \pi}{r^{2}} \xi^{2}(\delta)+\frac{4 \pi}{r} \delta \bar{g}(\delta) D A(\delta)
$$

We shall now show that at almost all $u f_{\delta} \rightarrow 0$ as $\delta \rightarrow 0$. The first term in $f_{\delta}$ tends to 0 at all $u$ as $\delta \rightarrow 0$. By Proposition 2, the second term tends to 0 at almost all $u$ as $\delta$ $\rightarrow 0$. It remains therefore to be shown that:

Lemma 8. At almost all $u, \delta D A(u, \delta) \rightarrow 0$ as $\delta \rightarrow 0$.

Proof. According to (5.27),

$$
D A(\delta)=\int_{\delta}^{\infty}\left[\frac{1}{2} g(h-\bar{h})-\xi\right](h-\bar{h}) \frac{d r}{r^{2}} .
$$

Thus

$$
|D A(\delta)| \leqq \frac{1}{2} \int_{\delta}^{\infty} g(h-\bar{h})^{2} \frac{d r}{r^{2}}+\int_{\delta}^{\infty}|\xi||h-\bar{h}| \frac{d r}{r^{2}}
$$


We decompose the first integral in (5.33) into

$$
\int_{\delta}^{\infty} g(h-\bar{h})^{2} \frac{d r}{r^{2}}=\int_{\delta}^{\delta^{1 / 2}} g(h-\bar{h})^{2} \frac{d r}{r^{2}}+\int_{\delta^{1 / 2}}^{\infty} g(h-\bar{h})^{2} \frac{d r}{r^{2}}
$$

(since we are interested only in the limit $\delta \rightarrow 0$, we can assume $\delta<1$ ). We estimate

$$
\delta \int_{\delta}^{\delta^{1 / 2}} g(h-\bar{h})^{2} \frac{d r}{r^{2}} \leqq \int_{\delta}^{\delta^{1 / 2}} g(h-\bar{h})^{2} \frac{d r}{r}=\frac{1}{4 \pi}\left(g\left(\delta^{1 / 2}\right)-g(\delta)\right) \rightarrow 0 \quad \text { as } \quad \delta \rightarrow 0,
$$

by the continuity of $g$ with respect to $r$ at $r=0$. Also,

$$
\delta \int_{\delta^{1 / 2}}^{\infty} g(h-\bar{h})^{2} \frac{d r}{r^{2}} \leqq \delta^{1 / 2} \int_{0}^{\infty} g(h-\bar{h})^{2} \frac{d r}{r}=\frac{\delta^{1 / 2}}{4 \pi}(1-g(0)) \rightarrow 0 \quad \text { as } \quad \delta \rightarrow 0 .
$$

We conclude that at all $u$,

$$
\delta \int_{\delta}^{\infty} g(h-\bar{h})^{2} \frac{d r}{r^{2}} \rightarrow 0 \quad \text { as } \quad \delta \rightarrow 0 .
$$

We decompose the second integral in (5.33) into

$$
\int_{\delta}^{\infty}|\xi||h-\bar{h}| \frac{d r}{r^{2}}=\int_{\delta}^{\delta^{1 / 2}}|\xi||h-\bar{h}| \frac{d r}{r^{2}}+\int_{\delta^{1 / 2}}^{1}|\xi||h-\bar{h}| \frac{d r}{r^{2}}+\int_{1}^{\infty}|\xi||h-\bar{h}| \frac{d r}{r^{2}} .
$$

We estimate

$$
\delta \int_{\delta}^{\delta^{1 / 2}}|\xi||h-\bar{h}| \frac{d r}{r^{2}} \leqq \int_{\delta}^{\delta^{1 / 2}}|\xi||h-\bar{h}| \frac{d r}{r} \leqq\left(\int_{\delta}^{\delta^{1 / 2}} \frac{1}{g} \frac{\xi^{2}}{r} d r\right)^{1 / 2}\left(\int_{\delta}^{\delta^{1 / 2}} g \frac{(h-\bar{h})^{2}}{r} d r\right)^{1 / 2} .
$$

Now, at almost all $u \xi / g^{1 / 2} r^{1 / 2} \in L^{2}\left(0, r_{0}\right), r_{0}$ arbitrary. Therefore at almost all $u$ the first factor in the above inequality is finite and tends to 0 as $\delta \rightarrow 0$. The second factor is equal to $\left(\left(g\left(\delta^{1 / 2}\right)-g(\delta)\right) / 4 \pi\right)^{1 / 2}$, which tends to 0 for all $u$ as $\delta \rightarrow 0$. Hence

$$
\delta \int_{\delta}^{\delta^{1 / 2}}|\xi||h-\bar{h}| \frac{d r}{r^{2}} \rightarrow 0
$$

as $\delta \rightarrow 0$, for almost all $u$. Also,

$$
\begin{aligned}
\delta \int_{\delta^{1 / 2}}^{1}|\xi||h-\bar{h}| \frac{d r}{r^{2}} & \leqq \delta^{1 / 2} \int_{0}^{1}|\xi| \frac{|h-\bar{h}|}{r} d r \leqq \delta^{1 / 2}\left(\int_{0}^{1} \frac{1}{g} \frac{\xi^{2}}{r} d r\right)^{1 / 2}\left(\int_{0}^{1} g(h-\bar{h})^{2} \frac{d r}{r}\right)^{1 / 2} \\
& \leqq\left(\frac{\delta}{4 \pi}\right)^{1 / 2}\left(\int_{0}^{1} \frac{1}{g} \frac{\xi^{2}}{r} d r\right)^{1 / 2} \rightarrow 0 \text { as } \delta \rightarrow 0,
\end{aligned}
$$

for almost all $u$. Furthermore, since we have $\int_{1}^{\infty}|h-\bar{h}| \frac{d r}{r^{2}} \leqq C$, where $C$ is a continuous function of $u$, the third integral in (5.36) is bounded by a multiple of $\sup |\xi|$, which, by Proposition 2, is finite for almost all $u$. We conclude that at almost all $u$

$$
\delta \int_{\delta}^{\infty}|\xi||h-\bar{h}| \frac{d r}{r^{2}} \rightarrow 0 \quad \text { as } \quad \delta \rightarrow 0 .
$$

Considering (5.35) and (5.37), together with (5.33), the lemma follows. 
Thus, for almost all $u$ and all $r>0, f_{\delta}(u, r) \rightarrow 0$ as $\delta \rightarrow 0$. Hence, from (5.31),

$$
\lim _{\delta \rightarrow 0} D\left(\frac{1}{r} \int_{\delta}^{r} g d r\right)=-\frac{1}{2 r} \bar{g}(g-\bar{g})-4 \pi \bar{g} D A+\frac{2 \pi}{r} \xi^{2} .
$$

For all $C^{\infty}$ functions $\phi$ whose support is compact and contained in the interior of $Q$, we have

$$
-\iint_{Q}\left(\frac{1}{r} \int_{\delta}^{r} g d r\right) D \phi d u d r=\iint_{Q} D\left(\frac{1}{r} \int_{\delta}^{r} g d r\right) \phi d u d r .
$$

As $\frac{1}{r} \int_{\delta}^{r} g d r \rightarrow \bar{g}$ for $\delta \rightarrow 0$ uniformly in the support of $\phi$, it follows that

$$
-\iint_{Q} \tilde{g} D \phi d u d r=\lim _{\delta \rightarrow 0} \iint_{Q} D\left(\frac{1}{r} \int_{\delta}^{r} g d r\right) \phi d u d r .
$$

Since $\phi f_{\delta}$ is dominated by an integrable function (see Proposition 2 and proof of Lemma 8 ) and tends to 0 almost everywhere as $\delta \rightarrow 0$, by the dominated convergence theorem, we have

$$
\lim _{\delta \rightarrow 0} \iint_{Q} D\left(\frac{1}{r} \int_{\delta} g d r\right) \phi d u d r=\iint_{Q} \lim _{\delta \rightarrow 0} D\left(\frac{1}{r} \int_{\delta}^{r} g d r\right) \phi d u d r .
$$

We conclude that $\bar{g}$ is weakly differentiable in $Q$ and

$$
D \bar{g}=\lim _{\delta \rightarrow 0} D\left(\frac{1}{r} \int_{\delta}^{r} g d r\right)=-\frac{1}{2 r} \bar{g}(g-\bar{g})-4 \pi \bar{g} D A+\frac{2 \pi}{r} \xi^{2} .
$$

It follows that $m:=(r / 2)(1-\bar{g} / g)$ is weakly differentiable in $Q$ and

$$
\begin{aligned}
D m= & -\frac{1}{4} \bar{g}\left(1-\frac{\bar{g}}{g}\right)-\frac{r}{2} \frac{1}{g} D \bar{g}+\frac{r}{2} \frac{1}{g^{2}} \bar{g} D g=-\frac{1}{4} g\left(1-\frac{\bar{g}}{g}\right) \\
& -\frac{r}{2} \frac{1}{g}\left[-\frac{1}{2 r} \bar{g}(g-\bar{g})-4 \pi \bar{g} D A+\frac{2 \pi}{r} \xi^{2}\right]-2 \pi r \frac{\bar{g}}{g} D A=-\frac{\pi}{g} \xi^{2} .
\end{aligned}
$$

We conclude that the mass equation is satisfied in $Q$. Thus (see [1, Sects. 2-4]) the complete system of Einstein's equations is satisfied in $Q$.

We now compute the evolution law of $\int_{\delta}^{r}(g / \bar{g}) d r, \delta>0$. We have

$$
\begin{aligned}
D \int_{\delta}^{r}(g / \bar{g}) d r & =\int_{\delta}^{r}\left(D(g / \bar{g})+\frac{1}{2} \bar{g} \frac{\partial}{\partial r}(g / \bar{g})\right) d r-\frac{1}{2} \bar{g}(g / \bar{g}) \\
& =\int_{\delta}^{r}\left(D(g / \bar{g})+\frac{1}{2} \frac{\partial g}{\partial r}-\frac{1}{2} \frac{g}{\bar{g}} \frac{\partial \bar{g}}{\partial r}\right) d r-\frac{1}{2} g \\
& =\int_{\delta}^{r}\left(D(g / \bar{g})-\frac{1}{2 r} \frac{g}{\bar{g}}(g-\bar{g})\right) d r-\frac{1}{2} g(\delta),
\end{aligned}
$$

and, since $g / \bar{g}=(1-2 m / r)^{-1}$, the mass equation gives,

$$
\begin{aligned}
D(g / \bar{g}) & =\left(1-\frac{2 m}{r}\right)^{-2}\left(\frac{2 D m}{r}+\frac{m}{r^{2}} \bar{g}\right)=\frac{2}{r}\left(\frac{g}{\bar{g}}\right)^{2} D m+\frac{1}{2 r} \frac{g}{\bar{g}}(g-\bar{g}) \\
& =-2 \pi \frac{g}{\tilde{g}^{2}} \frac{\xi^{2}}{r}+\frac{1}{2 r} \frac{g}{\bar{g}}(g-\bar{g}) .
\end{aligned}
$$


Hence we obtain

$$
D \int_{\delta}^{r}(g / \bar{g}) d r=-2 \pi \int_{\delta}^{r} \frac{g}{\bar{g}^{2}} \frac{\xi^{2}}{r} d r-\frac{1}{2} g(\delta),
$$

since it holds:

$$
\lim _{\delta \rightarrow 0} \int_{\delta}^{r}(g / \bar{g}) d r=\int_{0}^{r}(g / \bar{g}) d r
$$

and also

$$
\lim _{\delta \rightarrow 0} D \int_{\delta}^{r}(g / \bar{g}) d r=-2 \pi \int_{\delta}^{r} \frac{g}{\bar{g}^{2}} \frac{\xi^{2}}{r} d r-\frac{1}{2} g(0),
$$

we obtain by dominated convergence, in the same manner as in the preceding, that $\int_{0}^{r}(g / \bar{g}) d r$ is weakly differentiable in $Q$ and

$$
D \int_{0}^{r}(g / \bar{g}) d r=-2 \pi \int_{0}^{r} \frac{g}{\bar{g}^{2}} \frac{\xi^{2}}{r} d r-\frac{1}{2} g(0) .
$$

Integrating the mass equation along the characteristic $\chi_{u_{1}}\left(\cdot ; r_{1}\right)$ through $\left(u_{1}, r_{1}\right)$, we obtain the mass-flux relation

$$
m\left(u_{1}, r_{1}\right)+\pi \int_{0}^{u_{1}}\left[\frac{1}{g} \xi^{2}\right]_{\chi_{u_{1}}\left(; r_{1}\right)} d u=m\left(0, r_{0}\right),
$$

where $r_{0}:=\chi_{u_{1}}\left(0 ; r_{1}\right)$. Finally integrating $(5.42)$ along $\chi_{u_{1}}\left(\cdot ; r_{1}\right)$, we obtain the main integral identity:

$$
\int_{0}^{r_{1}} \frac{g}{\bar{g}}\left(u_{1}, r_{1}\right) d r+2 \pi \iint_{Q\left(u_{1}, r_{1}\right)} \frac{g \xi^{2}}{\bar{g}^{2} r} d r d u+\frac{1}{2} \int_{0}^{u_{1}} g(u, 0) d u=\int_{0}^{r_{0}} \frac{g}{\bar{g}}(0, r) d r,
$$

where

$$
Q\left(u_{1}, r_{1}\right):=\left\{(u, r) \mid 0<r<\chi_{u_{1}}\left(u ; r_{1}\right), 0<u<u_{1}\right\} .
$$

The proof of Theorem 1 is now complete.

\section{References}

1. Christodoulou, D.: The problem of a self-gravitating scalar field. Commun. Math. Phys. 105, 337-361 (1986)

2. Leray, J.: Sur le mouvement d'un liquide visquex emplissant l'espace. Acta Math. 63, 193-248 (1934)

Communicated by S.-T. Yau 
